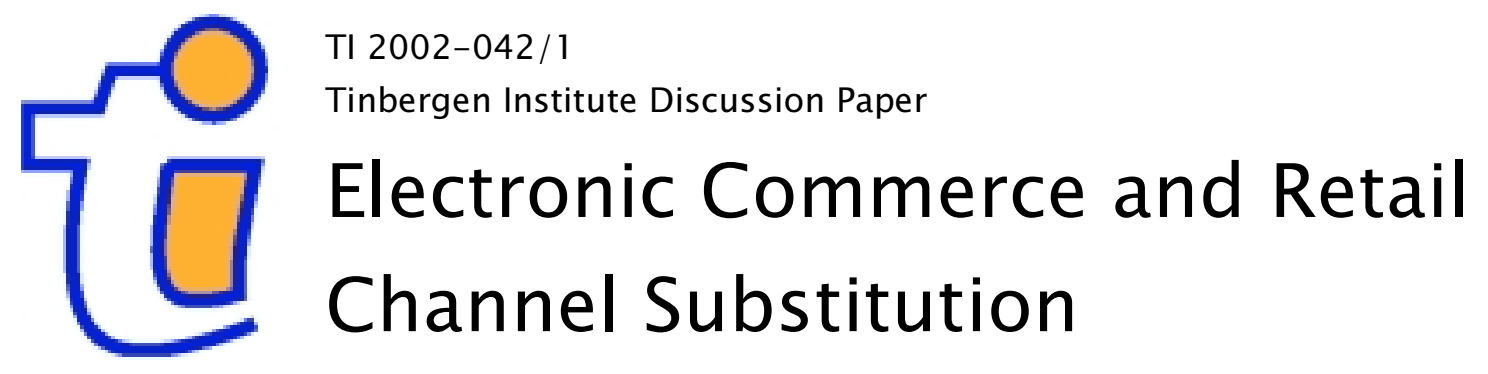

Maarten C.W. Janssen

Rob van der Noll

Department of Economics, Faculty of Economics, Erasmus University Rotterdam, and Tinbergen Institute 
Tinbergen Institute

The Tinbergen Institute is the institute for economic research of the Erasmus Universiteit Rotterdam, Universiteit van Amsterdam and

Vrije Universiteit Amsterdam.

Tinbergen I nstitute Amsterdam

Keizersgracht 482

1017 EG Amsterdam

The Netherlands

Tel.: +31.(0)20.5513500

Fax: $\quad+31 .(0) 20.5513555$

Tinbergen Institute Rotterdam

Burg. Oudlaan 50

3062 PA Rotterdam

The Netherlands

Tel.: $\quad+31 .(0) 10.4088900$

Fax: $\quad+31 .(0) 10.4089031$

Most TI discussion papers can be downloaded at

http://www.tinbergen.nl 


\title{
Electronic Commerce and Retail Channel Substitution*
}

\author{
Maarten C.W. Janssen ${ }^{\dagger}$ and Rob van der Noll ${ }^{\ddagger}$ \\ Erasmus University Rotterdam and Tinbergen Institute
}

April 2002

\begin{abstract}
We analyze a market where firms compete in a conventional and an electronic retail channel. Consumers easily compare prices online, but some incur purchase uncertainties on the online channel. We investigate the market shares of the two retail channels and the prices that are charged. We find that the share of the electronic channel is decreasing in the size of the uncertainty. Furthermore, searching consumers do not always buy. They drop out when the uncertainty associated with buying online is not offset by a low price. Finally, the model exhibits price dispersion and the expected price is increasing in the magnitude of the online purchase uncertainty.
\end{abstract}

${ }^{*}$ This version substitutes a previous version of the paper, entitled 'E-commerce and channel substitution for differentiated products'. We thank the participants of The Econometric Society European Meeting 2001, The European Economic Association Congress 2001, the TIR lunchseminar, ASSET Conference 2001 and the participants of the 4th Internet Economics Workshop in Berlin for useful comments. We thank Vladimir Karamychev for programming assistance.

${ }^{\dagger}$ Dept. of Economics, Erasmus University Rotterdam, P.O. Box 1738, 3000 DR Rotterdam, The Netherlands.

$¥$ Tinbergen Institute, Burg. Oudlaan 50, 3062 PA Rotterdam, The Netherlands. Phone: +3110 4088901, Fax: +31 10 4089031, E-mail: vandernoll@few.eur.nl. 


\section{Introduction}

The importance of the Internet as a marketplace has substantially grown the past few years, even though expectations have tempered after March 2000. A distinctive feature of doing business electronically is that transactions no longer require the physical coordination of buyers and sellers: market participants find each other at their screens. There are many aspects of market interaction which are affected by this online nature of trade. On the supply side, we may think of all kinds of cost reductions, resulting from new ways of organizing production and sales processes. On the demand side, the major impact of the Internet is on consumers' ability to acquire information about product characteristics and prices. Moreover, due to the global nature of the Internet, there is a potentially dramatic increase in market size: a firm selling online may reach customers worldwide, round the clock. Finally, as far as information goods are concerned, the distribution process can be made entirely electronically.

While electronic commerce has an enormous potential, it is good to realize that even for books and CDs it is still of minor importance compared to other retail channels. ${ }^{1}$ It is important, however, to consider the question to what extent consumers will substitute the Internet for the conventional retail channel. This paper provides a theoretical framework that sheds some light on the relative importance of different factors that determine the market share of electronic sales. In doing so, we also investigate the way in which the Internet may promote competition in markets.

The basic point of our paper is to account for the relative strengths and weaknesses of the online retail channel compared to conventional stores. On the Internet, it is easy to find firms and compare their prices, but the online nature of the transaction leads to uncertainties. For example, it is more difficult to assess how well a particular product fits the specific needs of the costumer. Other uncertainties and inconveniences are related to the payment method and the delivery. ${ }^{2}$ We refer to the uncertainty associated with an online purchase as 'online uncertainty'. In a conventional store, physical inspection of goods is much easier, but in order to compare firms' offers one has to make more than one costly visit. We will refer to this disadvantage of a visit in the offline channel as 'best-price uncertainty'. It is therefore the purpose of this paper to contribute to the understanding of the functioning of electronic markets by analyzing the trade-off between (i) online uncertainty on the Internet and (ii) best-price uncertainty in the conventional store.

The view that some consumers may derive a lower value from an online purchase,

\footnotetext{
${ }^{1}$ According to a study by the Boston Consulting Group (2000) in the United States and Europe, e-commerce accounts for less than 5 percent of total sales in the books and CDs categories.

${ }^{2}$ A survey by Taylor Nelson Sofres (2001) indicates that the most important reason for not purchasing online is related to payment security and the second most important reason is given by 'You don't know what you get.' and 'Easier/more fun to buy goods/services in a store.'
} 
as compared to a conventional purchase, can be expressed as follows:

"Why do users decide not to shop?

The reasons why more online users decide not to shop online despite the fact that they have considered it, than those who actually do, may be several: ... E-commerce web sites must also carry part of the responsibility, as many are not good at making the shopping experience easy and trustworthy for the online users. Lack of privacy statements, difficult navigation, poor product declarations, slow delivery of goods, limited price discounts, uncertainty around security, and unclear redemption policies are examples of issues faced by the users." Taylor Nelson Sofres (2000).

We present a duopoly model where firms sell in both the conventional and the electronic retail channel. Firms sell homogeneous products and the price at which a firm sells the product is the same in both retail channels. There are two types of consumers. Experienced consumers do not incur purchase uncertainties online. As a result they always search on the Internet for the lowest price of the product. Inexperienced consumers, in contrast, incur an online uncertainty. For example, they do not know which version of a product they like best, prior to physical inspection. They therefore face a trade-off between best-price uncertainty, if they purchase in the store, and online uncertainty, if they purchase online. We shall refer to the percentage of experienced consumers in the economy as market maturity. The pricing decisions by firms and the shopping decisions by buyers are modelled as a simultaneous move game.

By means of this model, we arrive at the following insights. First, searching consumers do not always buy. We find that inexperienced consumers who search the Internet may not purchase, if the online uncertainty is not compensated by a low price. As was pointed out above by Taylor, Nelson and Sofres, this so-called drop-out seems relevant for electronic markets.

Second, the share of the market captured by the electronic channel crucially depends on the importance of the online uncertainty and the maturity of the market. The more non-digital product attributes matter, such as in the clothing industry, the more important is physical inspection, hence the online uncertainty is large in that case. As a result, more buyers frequent the conventional store and channel substitution is small. Market maturity has the following surprising impact: if some inexperienced consumers use the Internet, the total market share of this channel decreases in market maturity. The reason for this result is that when a market matures, the gain from comparing prices decreases.

Third, we show that the equilibrium exhibits price dispersion. The reason for this result is as follows. Firms have monopoly power over those consumers who 
visit the physical store and observe only one price. This gives firms incentives to charge high prices. However, firms also aim at attracting the consumers who browse their Internet homepages searching for the best price. These consumers give firms incentives to charge lower prices. The interplay of these two forces results in price dispersion: some firms aim at selling to a few consumers in the conventional store at higher prices, others aim at selling to more consumers at lower prices. In equilibrium, the two different strategies result in the same profit.

Fourth, the equilibrium price distribution is such that marginal cost pricing never occurs. Interestingly, the expected price increases in the number of price comparing consumers, if some inexperienced consumers also use the Internet. Finally, if no inexperienced consumer uses the Internet, the price distribution is not depending on online uncertainty, while if this channel does receive some inexperienced consumers, the expected price is increasing in online uncertainty.

Our theoretical framework is built around three crucial assumptions. Firstly, both firms are present on both retail channels. The relevance of these so-called multichannel retailers has widely been recognized. In Europe, multichannel retailers accounted for two-thirds of the online retail sales in 1999 (Boston Consulting Group 2000). A 'clicks-and-mortar' approach seems to be good business practice, as multichannel retailers have a brand advantage over their 'pure-play' counterparts. Internet retailers start recognizing the importance of a physical presence for customer satisfaction. The two other other important features of our model are that, for a particular product, a firm sets the same price on the Internet as in the physical store, and that consumers search only once. Section 5 discusses these assumptions extensively.

There is an emerging theoretical and empirical literature on the implications of the Internet for the competitiveness of markets. On the empirical side, a number of studies compare average prices and price dispersion in electronic marketplaces against those in conventional markets. Some studies find that prices in electronic markets are lower than corresponding prices in conventional markets (see, e.g., Brynjolfsson and Smith (1999)), while others report that prices in electronic markets are approximately equal to (see, e.g., Clay et al. (2000)) or even higher than prices in conventional stores (see, e.g., Lee (1997) and Bailey (1998)). Some of these studies also find that price dispersion is not lower on-line than in traditional outlets.

On the theoretical side, there are papers by Bakos (1997) and Janssen and Moraga (2000), among others, studying the implications of a reduction in search cost due to the emergence of Internet on the competitiveness of markets. Bakos presents a model of circular product differentiation where consumers search for prices and product features. In his model, consumers can get to know all product characteristics if they engage in costly search. In our model, in contrast, consumers cannot get around some of the uncertainties associated with buying on the Internet. Commenting on Bakos' 
paper, Harrington, Jr. (2001) questions the validity of some of Bakos' results.

Janssen and Moraga present a consumer search model showing that a reduction in consumer search cost does not unambiguously imply increased competition.

There are a few papers addressing the issue of channel substitution. Zettelmeyer (2000) focuses on the incentives firms have to increase the ease with which consumers can evaluate their products. Consumer search cost is in this way a strategic variable of firms. He shows that firms may provide selected groups of consumers with different information (level of search cost), thereby providing monopoly power to the firms and reducing market competition. The focus of our model is different. The source of monopoly power in our model lies in the fact that some consumers visit one conventional store only and, hence, do not compare prices. In other words, a major difference is that in our model consumers have to search to learn prices, whereas prices are known in Zettelmeyer's world.

Lal and Sarvary (1999) pose a different question: When is the Internet likely to decrease price competition? They study a model in which a consumer needs to evaluate the product, like clothing, physically to learn about its non-digital attributes. Firms are also present on two retail channels. Since digital attributes can be communicated over the web, the Internet changes the effective search cost structure: without the Internet, the cost of evaluating an unfamiliar brand of clothing is the cost of visiting an additional store. With the Internet however, consumers do not have to visit the store at all to buy their familiar brand, and thus, the cost of trying another brand is to undertake the entire shopping trip. In this way, the Internet may increase the effective cost of search and customer loyalty, which decreases price competition.

Finally, Mazón and Pereira (2000) analyse whether firms have incentives to open electronic retail channels and the different price equilibria these incentives generate. They show that, depending on variables as possible retail cost reductions, established firms may or may not compete with new virtual shops.

The paper is organized as follows. Section 2 presents the basic model. Section 3 analyzes the equilibrium properties. Section 4 discusses the model's predictions when parameters values change. A discussion about the robustness of the model and conclusions are contained in Section 5 and proofs are contained in an Appendix.

\section{The Model}

On the supply side there are two vertically integrated firms, each offering a homogeneous good in a conventional physical store and on the Internet. Firms produce the good at constant returns to scale at zero production cost.

On the demand side of the market, there is a mass of consumers, normalised to one. The consumer has a maximum willingness to pay for the good equal to $v$ and 
purchases at most one unit. There are two types of consumers. The first type incurs an online uncertainty such that when she buys online, the value of her purchase is equal to $\lambda v$, where $\lambda \leq 1$. We will refer to this type as the inexperienced consumer. The second type does not incur this uncertainty. We will call this second type the experienced consumer. The uncertainty can be seen as resulting from the inability to physically inspect a good online and hence, to select the most preferred version of a good. In this interpretation, the novel feature is that some consumers do not know what they want to have prior to inspection, that is, they are uncertain about their preferences, while some do know their preferences. ${ }^{3}$

There are $1-\mu$ inexperienced consumers and $\mu$ experienced consumers, with $0 \leq \mu \leq 1$. Both types have a restricted time endowment such that consumers can either visit the Internet, where they can observe all prices, or they can visit one store. ${ }^{4}$

In our model, firms and consumers play a simultaneous move game. An individual firm $i$ sets its price $p^{i}$, taking price behavior of the rival, firm $j$, as well as consumers' search behavior as given. Consumers form expectations about their individual valuations and the prices in the market and decide which channel to search. Experienced consumers do not incur online uncertainty and they will therefore just look for the best price. Inexperienced consumers however face a trade-off and may either search the store or the Internet. Specifically, we will say that a fraction $\alpha$ searches the store and a fraction $(1-\alpha)$ searches the Internet, with $0 \leq \alpha \leq 1$. If this consumer searches the Internet, she either buys at the lowest price, or if this lowest price is too high, she exits without making a purchase. If she searches the conventional store, she observes only the price charged by the particular firm she visits.

The pay-offs to the players of this game can be expressed as follows. For the firms:

$$
\Pi^{i}\left(p^{i}, p^{j}\right)= \begin{cases}{\left[\frac{\alpha(1-\mu)}{2}+\mu+(1-\alpha)(1-\mu)\right] p^{i}} & \text { if } \quad p^{i}<p^{j} \text { and } p^{i} \leq \lambda v \\ \left.\frac{\alpha(1-\mu)}{2}+\mu\right] p^{i} & \text { if } \quad p^{i}<p^{j} \text { and } \lambda v<p^{i} \leq v \\ {\left[\frac{\alpha(1-\mu)}{2}\right] p^{i}} & \text { if } \quad p^{j}<p^{i} \leq v\end{cases}
$$

This can be understood as follows. Firm $i$ obtains a per consumer profit of $p^{i}$. In the store, the firm sells to the inexperienced consumers who $(i)$ search the store, which happens with probability $\alpha$ and $(i i)$ who find the store of $i$, which happens with probability one half. This results in a demand of $\alpha(1-\mu) / 2$. On the Internet, the firm attracts all $\mu$ experienced consumers when it asks a lower price than the

\footnotetext{
${ }^{3}$ As mentioned above, another model that comes close to this feature is Zettelmeyer (2000). Above we discussed the major differences.

${ }^{4}$ Here we recognize that, although often suggested otherwise, searching on the Internet does take time. For simplicity, and without loss of generality, we assume that it takes the same amount of time to visit two sites electronically or one shop physically. Section 5 contains more discussion on this point.
} 
rival, since they observe all prices and buy from the cheapest firm. Additionally, the $(1-\alpha)(1-\mu)$ inexperienced consumers who search the Internet, also go for the lowest price, but purchase only if the lowest price does not exceed their maximum willingness to pay of $\lambda v$.

The expected value a consumer derives is straightforward to determine. For the inexperienced consumer:

$$
\begin{aligned}
& v-E\left(p^{i}\right) \\
& \operatorname{Pr}\left[\min \left[p^{1}, p^{2}\right] \leq \lambda v\right] \times \\
& \left\{\lambda v-E\left(\min \left[p^{1}, p^{2}\right] \mid \min \left[p^{1}, p^{2}\right] \leq \lambda v\right)\right\} \quad \text { if she searches the store of firm } i,
\end{aligned}
$$

The surplus for the experienced consumer can be described as follows:

$$
\begin{array}{ll}
v-E\left(\min \left[p^{i}, p^{j}\right]\right) & \text { if he searches the Internet, } \\
v-E\left(p^{i}\right) & \text { if he searches the store of firm } i .
\end{array}
$$

Since we will illustrate below that the model exhibits price dispersion, we have $E(p)>E(\min [p])$. It is then easy to see that the experienced consumer will choose to visit the Internet, since he can obtain the same value $v$ in both channels.

\section{Equilibrium Analysis}

In this section we will analyze possible equilibrium configurations and their properties. An equilibrium is a pricing strategy, one for each firm, and a set of decisions where to shop, one for each consumer, such that no one has an incentive to change his behavior. A first observation is that there is no pure strategy equilibrium. To see why, suppose firm $i$ adopts the strategy to set $p^{i *}$, its rival will have an incentive to set its price just below $p^{i *}$, such that it can capture all experienced consumers. If we continue this reasoning we end up with Bertrand prices, $p^{i *}=p^{j *}=0$. However, these prices do not constitute an equilibrium either: firm $i$ will raise price so as to collect some revenues from the inexperienced consumers in its store. ${ }^{5}$

Thus, firms play mixed strategies and randomly pick a price from a set of prices, with each price generating the same expected pay-off. We will denote the mixed strategy of firms by $F(p)$ : the probability that a firm charges a price smaller than $p$.

The following result shows that there are no equilibria in which all inexperienced consumers prefer the Internet, i.e. $\alpha=0$. Therefore, full retail channel substitution does not occur. Additionally, we have shown above that experienced consumers never

\footnotetext{
${ }^{5} \mathrm{~A}$ similar argument establishes that there are no mass points in the mixed strategy of firms.
} 
use the physical store. The lemma proves.

Lemma 1 An equilibrium where (i) $\alpha=0$ or (ii) experienced consumers use the physical store does not exist.

Proof. (i) Suppose $\alpha=0$, then all consumers of both types would use the Internet and therefore, observe all prices. This would lead firms to charge Bertrand prices, i.e. both prices equal to marginal cost, normalized to zero $p^{i}=p^{j}=0$. If this were so, inexperienced consumers would prefer the store, since there they can avoid online inconveniences, and hence, make a better purchase. Thus, $\alpha=0$ cannot be part of an equilibrium.

(ii) Experienced consumers obtain valuation $v$ in both channels, but pay a strictly lower price on the Internet than in the store, since $E\left(\min \left[p^{i}, p^{j}\right]\right)<E(p){ }^{6}$

We now proceed by investigating the pricing behavior of firms for the case that $\alpha>$ 0 . We will distinguish two situations: $\alpha=1$, i.e., all inexperienced consumers visit the store and hence, no channel substitution occurs, and $0<\alpha<1$, i.e., inexperienced consumers randomize between visiting the store and visiting the Internet. This second case can be interpreted as a situation of partial channel substitution, with some inexperienced consumers visiting the store, while others visit the Internet.

\subsection{No channel substitution}

In this case all inexperienced consumers use the conventional channel, i.e. $\alpha=1$, and all experienced consumers use the Internet. By substituting $\alpha=1$ in (1) we can write expected profits as:

$$
\Pi^{i}\left(p^{i}, F(p)\right)=\left[\frac{(1-\mu)}{2}+\mu\left(1-F\left(p^{i}\right)\right)\right] p^{i},
$$

where $\left(1-F\left(p^{i}\right)\right)$ represents the probability that $i$ is the cheapest firm.

Firms are indifferent between all prices in a certain support. It is easily seen that the upperbound of the price support equals $v$. Charging prices higher than $v$ cannot be optimal as no consumer will buy. Also, by charging a price equal to the upperbound, a firm will only make profits from consumers who visit the shop. But, if the highest price a firm would ever charge is strictly smaller than $v$, then the firm could raise profits on these consumers by charging higher prices. Hence, the highest price a firm will ever charge equals $v$ and the profit the firm makes in this case is

\footnotetext{
${ }^{6}$ The combination of pure strategies in which firms charge the monopoly price $H$ and both types of consumers use the store can be disregarded. As it is well known in the search literature, this equilibrium has the informed (here: experienced) consumer playing a weakly dominated strategy.
} 
$\frac{(1-\mu)}{2} v$. Since the firm must be indifferent between setting any price in the support of $F$, the following equality must hold:

$$
\Pi^{i}(\cdot)=\left[\frac{(1-\mu)}{2}+\mu\left(1-F\left(p^{i}\right)\right)\right] p^{i}=\frac{(1-\mu)}{2} v .
$$

By rewriting this equality, we can find the mixed strategy price distribution ${ }^{7}$

$$
F(p)=\frac{1+\mu}{2 \mu}-\frac{1-\mu}{2 \mu} \frac{v}{p}
$$

Now we want to investigate whether the proposed pricing behavior by firms and search behavior by consumers indeed constitutes an equilibrium. To this end, we now verify when the inexperienced consumer indeed will find it optimal to use the conventional channel. If she visits the physical shop, she will obtain her maximum willingness to pay, equal to $v$, but she is not able to make a price comparison between firms. In contrast, if she decides to browse the Internet, she will obtain only $\lambda v$ and buy at the best price if this price is low enough. Therefore, to let the physical store be the optimal channel for the inexperienced consumer, the following has to hold:

$$
\begin{aligned}
v-E(p) \geq & \operatorname{Pr}\left[\min \left[p^{1}, p^{2}\right] \leq \lambda v\right] \times \\
& \left\{\lambda v-E\left(\min \left[p^{1}, p^{2}\right] \mid \min \left[p^{1}, p^{2}\right] \leq \lambda v\right)\right\}
\end{aligned}
$$

which easily follows from (2). Since $v$ drops out of the above expression and the parameter $\mu$ enters via the expected price and minimum price, the conventional channel is indeed optimal for the inexperienced consumer for values of $\lambda$ and $\mu$ satisfying inequality (4), see the Appendix for a detailed derivation. In that case there exist equilibria that exhibit no channel substitution, in the sense that all inexperienced consumers will shop at the conventional store.

When the online uncertainty is high, $\lambda$ is low. Now the following Proposition states that for $\lambda$ low enough, the trade-off between online uncertainty and best-price uncertainty is made in favor of the store. Put differently, for low $\lambda$, we have that physical aspects are more important than price comparisons, no matter what the value of $\mu$ is. Additionally, for low $\lambda$, the probability of a purchase on the Net is lower, since the best price is less likely to be low enough. Thus, for $\lambda$ low enough (4) may be satisfied.

The result also states that (4) will be satisfied for $\mu$ low or high enough. This can be understood as follows. The difference between the prices the duopolists set, approaches zero for $\mu$ close to zero or close to one. Intuitively this makes sense,

\footnotetext{
${ }^{7}$ There must be some $\underline{p}$ for which $F(\underline{p})=0$, from (3) we obtain that the lowerbound of the support is $\underline{p}=\frac{(1-\mu) H}{1+\mu}$.
} 
for when everybody is experienced $(\mu=1)$ and buys on the Internet, competition is strong and Bertrand prices result. Similarly, when everybody is inexperienced $(\mu=0)$, monopoly prices may result and there is no price difference to be expected either. If the price difference is small, inexperienced consumers have no reason to search the Internet. Thus for $\mu$ small or large enough, we may have an equilibrium where inexperienced consumers buy in the store. The proposition summarizes our findings.

Proposition 2 (no channel substitution) An equilibrium of the game described above in which inexperienced consumers do not use the Internet and all experienced consumers do use the Internet exists when inequality (4) is met. Firms randomly select prices from the set $\left[\frac{(1-\mu) v}{1+\mu}, v\right]$ according to the cumulative distribution function $F(p)$ given by (3). Furthermore, there exists $\bar{\lambda}$ such that:

(i) if $\lambda \leq \bar{\lambda}$ the no channel substitution equilibrium exists for all $\mu$;

(ii) if $\lambda>\bar{\lambda}$ there exist $\underline{\mu}(\lambda)$ and $\bar{\mu}(\lambda)$ with $\underline{\mu}(\lambda)<\bar{\mu}(\lambda)$ such that the no channel substitution equilibrium exists for all $\mu<\underline{\mu}(\lambda)$ and all $\mu>\bar{\mu}(\lambda)$. Moreover, $\lim _{\lambda \rightarrow 1}$ $\underline{\mu}(\lambda)=0$ and $\lim _{\lambda \rightarrow 1} \bar{\mu}(\lambda)=1$.

\subsection{Partial channel substitution}

When inequality (4) is not satisfied, inexperienced consumers do not prefer the store, implying some buyers substitute the online for the offline retail channel. In this case, $\alpha \in(0,1)$ and the profits per firm are as in (1). Observe that if an inexperienced consumer chooses the Internet, her maximum willingness to pay is $\lambda v$ and this leads to a discontinuity of demand at $\lambda v$. The derivation of firm's behavior must account for this discontinuity. Since we have shown that there is no equilibrium in pure strategies, we will investigate how a mixed strategy can be described. Firstly, observe that for a given $\widehat{\alpha}$, firms will never be indifferent between a price slightly above $\lambda v$ and a price of $\lambda v$, since they prefer a price of $\lambda v$ in order to attract also the mass of $(1-\widehat{\alpha})(1-\mu)>0$ inexperienced consumers. Therefore, in equilibrium, prices in a right neighbourhood of $\lambda v$ will never be charged. Hence, the price support can be of one of the following forms:

1. the lowerbound of the price support is larger than or equal to $\lambda v$;

2. the upperbound of the price support is smaller than or equal to $\lambda v$;

3. the support is nonconvex and contains a 'hole': prices not greater than $\lambda v$ are charged and prices strictly higher than $\lambda v$ are charged, leaving open an interval of prices that will not be set in equilibrium. 
The first case implies that $\alpha=1$ as illustrated in Section 3.1: since the probability that the best price is low enough is zero, no inexperienced consumer will search the Internet. The analysis is provided above. The second case cannot be an equilibrium since in this case, charging a price equal to $v$ dominates charging the upperbound.

Therefore, for an equilibrium with $\alpha<1$, the price support must be as described in the third case. In equilibrium, prices in a certain range are charged with zero probability. For convenience, we denote by $x \equiv \alpha(1-\mu)$ the share of all consumers going to the shop. Hence, $x$ is the mass of consumers on which firms exercise market power. In the Appendix, we show that

Lemma 3 In equilibrium, prices in the interval $\left(\lambda v, \frac{(1-x) v}{1-x+\frac{\mu(1-\lambda)}{\lambda}}\right)$ are set with zero probability.

Let us denote the upperbound of this interval by $p^{\prime} \equiv \frac{(1-x) v}{1-x+\frac{\mu(1-\lambda)}{\lambda}}$. Now we want to describe the cumulative distribution function for prices above $p^{\prime}$, denoted $F^{u}(p)$, and for prices below $\lambda v$, denoted $F^{l}(p)$. For $p \geq p^{\prime}$, the following must hold:

$$
\left(\frac{x}{2}+\mu\left[1-F^{u}(p)\right]\right) p=\frac{x}{2} v
$$

since, as was argued in Section 3.1, the maximum price a firm will charge is $p=v$ and at any price in the support of $F$, expected profits must equal profits at $p=v$. It follows that:

$$
F^{u}(p)=\frac{2 \mu+x}{2 \mu}-\frac{x}{2 \mu} \frac{v}{p}
$$

For prices $p \leq \lambda v$, we must have that

$$
\left(\frac{x}{2}+[1-x]\left[1-F^{l}(p)\right]\right) p=\frac{x}{2} v
$$

and we obtain:

$$
F^{l}(p)=\frac{2-x}{2-2 x}-\frac{x}{2-2 x} \frac{v}{p}
$$

Summarizing, we can describe the strategy of the firm as follows: ${ }^{8}$

$$
F(p)= \begin{cases}\frac{2-x}{2-2 x}-\frac{x}{2-2 x} \frac{v}{p} & \text { if } \underline{p} \leq p \leq \lambda v \\ 1-\frac{x(1-\lambda)}{2 \lambda(1-x)} & \text { if } \lambda v \leq p \leq p^{\prime} \\ \frac{2 \mu+x}{2 \mu}-\frac{x}{2 \mu} \frac{v}{p} & \text { if } p^{\prime}<p \leq v\end{cases}
$$

\footnotetext{
${ }^{8}$ To find the lowerbound of the support, we solve $\frac{2-x}{2-2 x}-\frac{x}{2-2 x} \frac{H}{p}=0$, resulting in $\underline{p}=\frac{x H}{2-x}$.
} 
Note that when $\alpha=1$, the 'hole' disappears and the case of no channel substitution analyzed above emerges.

To find an equilibrium, we must now turn to the demand side to describe how $\alpha$ is determined. Since $\alpha \in(0,1)$ the consumer must be indifferent between the two retail channels. The following equality must therefore be satisfied:

$$
\begin{aligned}
v-E(p)= & \operatorname{Pr}\left[\min \left[p^{1}, p^{2}\right] \leq \lambda v\right] \times \\
& \left\{\lambda v-E\left(\min \left[p^{1}, p^{2}\right] \mid \min \left[p^{1}, p^{2}\right] \leq \lambda v\right)\right\} .
\end{aligned}
$$

The above expression therefore implicitly determines $\alpha$. The left-hand side of this expression represents the expected surplus of visiting the physical shop and the right-hand side represents the expected surplus of visiting the Internet. We let $\pi \equiv \operatorname{Pr}\left[\min \left[p^{1}, p^{2}\right] \leq \lambda v\right]$ denote the probability of purchase for an online inexperienced consumer. ${ }^{9}$

To obtain the expression above explicitly, we derive the expected price and the conditional expected minimum price. In the Appendix the reader can check that

Lemma 4 The expected price is given by

$$
E(p)=\frac{x v}{2(1-x)} \ln \left[\frac{\lambda(2-x)}{x}\right]+\frac{x v}{2 \mu} \ln \left[1+\frac{(1-\lambda) \mu}{\lambda(1-x)}\right]
$$

and the conditional expected minimum price is given by $y^{10}$

$$
\begin{array}{r}
E\left(\min \left[p^{i}, p^{j}\right] \mid \min \left[p^{i}, p^{j}\right] \leq \lambda v\right)=\frac{1}{\pi} \frac{x^{2} v}{2(1-x)^{2}}\left[\frac{-1}{\lambda}+\frac{2-x}{x}-\ln \left[\frac{(2-x) \lambda}{x}\right]\right] \\
\text { for } 0 \leq x \leq \bar{x} \equiv \frac{2 \lambda}{\lambda+1} .
\end{array}
$$

Now that we have determined the expected price and the relevant minimum price, we consider again the equation that determines $\alpha$. Rewriting the equilibrium condition given by $(6)$, we define $\Gamma(x ; \lambda, \mu)$ by

$$
\Gamma(x ; \lambda, \mu)=\pi\left\{\lambda v-E\left(\min \left[p^{1}, p^{2}\right] \mid \min \left[p^{1}, p^{2}\right] \leq \lambda v\right)\right\}+E(p)-v .
$$

The value of $\Gamma(x ; \lambda, \mu)$ represents the surplus that can be obtained by visiting the Internet, in excess of the surplus in the store. Therefore, $\Gamma(x ; \lambda, \mu)>0$ implies that

\footnotetext{
${ }^{9}$ Consumers might be tempted to continue searching and visit the store after the search on the Internet. We discuss this possibility in Section 5.

${ }^{10}$ Note that the conditional expected minimum price is defined up to a certain upperbound on $x$. This restriction on the domain of $x$ arises because for high enough $x$, the lowerbound of the price support exceeds $\lambda H$. Below we will discuss this in a more intuitive way.
} 


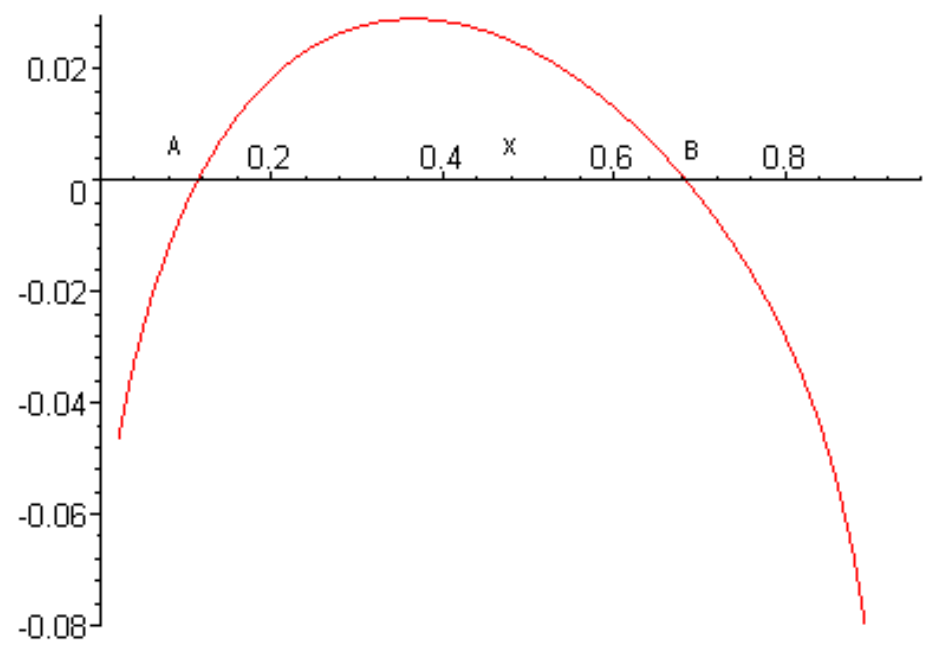

Figure 1: The equilibrium condition $\Gamma(x ; .93, .5)$ for $0<x<\bar{x} . \Gamma(\cdot)>0$ implies consumers have an incentive to search the Internet. As $x \equiv \alpha(1-\mu)$, the function is in this case defined only for $0 \leq x \leq 0.5$.

consumers prefer the Internet and $\Gamma(x ; \lambda, \mu)<0$ implies that they prefer the physical store. By employing Lemma 4 we can write this function explicitly as:

$$
\begin{aligned}
\Gamma(x ; \lambda, \mu)=\frac{x}{(1-x)^{2}}\left(\frac{x}{4}(\right. & \left.\left.4-\lambda+\frac{1}{\lambda}\right)-1\right)+\frac{x}{2(1-x)^{2}} \ln \frac{\lambda(2-x)}{x}+ \\
& +\frac{x}{2 \mu} \ln \left[1+\frac{(1-\lambda) \mu}{\lambda(1-x)}\right]-1+\lambda=0, \text { for } 0 \leq x \leq \bar{x} .
\end{aligned}
$$

For any given $\lambda$ and $\mu$, this equality determines the equilibrium value of $\alpha \in(0,1)$ if it exists. In the Appendix we prove the following useful properties:

Fact $1 \Gamma(0 ; \lambda, \mu)<0$ for all $\lambda<1$ and all $\mu ; \Gamma(0 ; 1, \mu)=0$ for all $\mu$.

Fact $2 \Gamma(\bar{x} ; \lambda, \mu)<0$ for all $\mu>0$ and all $\lambda<1 ; \Gamma(\bar{x} ; \lambda, 0)=0$ for all $\lambda$.

Fact $\left.3 \frac{\partial \Gamma(x ; \lambda, \mu)}{\partial x}\right|_{x=0}>0$.

Fact 4 There exist at most two values of $x \in[0, \bar{x}]$ such that $\Gamma(x ; \lambda, \mu)=0$.

Fact $\left.5 \frac{\partial^{2} \Gamma(x ; \lambda, \mu)}{\partial x^{2}}\right|_{x=0}<0$.

By using these facts, we may plot $\Gamma(x ; \lambda, \mu)$ as a function of $x$ as in Figure (1). ${ }^{11}$ The first three facts and the shape of this curve can be understood as follows. Recall

\footnotetext{
${ }^{11}$ In the Figure we have $\frac{\partial^{2} \Gamma(\cdot)}{\partial x^{2}}<0$ but this is not in general the case.
} 
that $\Gamma(x ; \lambda, \mu)>0$ implies that consumers prefer the Internet and $\Gamma(x ; \lambda, \mu)<0$ implies that they prefer the physical store. An alternative interpretation is that the curve shows how beneficial it is to compare prices. Furthermore, $x$ represents the segment of the market over which firms exercise market power. Now when $x=0$, firms only sell to price comparing buyers and are induced to price competitively. There is no benefit to comparing prices and inexperienced consumers prefer to avoid online inconveniences and go to the physical shop. This is reflected in Fact 1 . As $x$ increases, we see that $\Gamma(x ; \lambda, \mu)$ first increases. This is because it becomes more beneficial to compare prices, when firms gain market power and price dispersion emerges. Hence, it becomes more beneficial to search the Internet. Fact 3 states this formally. For $x$ sufficiently large, we see that $\Gamma(x ; \lambda, \mu)$ starts to decrease. This can be explained by the fact that when firms have a lot of market power, they price closer to the monopoly price, and then the price dispersion decreases in $x$. Additionally, as firms price closer to the monopoly price, it becomes less likely that the best price is low enough. Hence, comparing prices on the Internet becomes less rational. Finally, as $x$ exceeds $\bar{x}, \pi$ becomes zero. The surplus of buying on the Internet becomes zero as the consumer expects that even the lowest of the two prices she will observe will be too high. This is given by Fact 2 .

For an equilibrium in which both retail channels receive some inexperienced consumers, it must be that $\Gamma(x ; \lambda, \mu)=0$. Since $x \in(0,1-\mu]$ we may infer that for small $\mu$, the curve is first increasing and then decreasing. Hence, there may be two intersection points of the line with the horizontal axis. It may be questioned whether both these points reflect stable equilibria.

To address the issue of stability, recall that $\Gamma(x ; \lambda, \mu)$ represents the surplus that can be obtained in excess of the surplus attainable in the store. Consider now the two points of intersection A and B in Figure (1). To the right of point B, consumers expect to derive more value from searching the store than from searching the Internet. They will therefore prefer the store so that $\alpha$ (and $x$ ) has a tendency to rise. Between points $\mathrm{A}$ and $\mathrm{B}$, the consumer derives more value from searching the Internet than from searching the store. This is because the expected price difference is high. Consumers will prefer to search the store less intensely, i.e., $\alpha$ (and $x$ ) decreases. A similar argument establishes that to the left of point $\mathrm{A}, \alpha$ has a tendency to increase. Point $\mathrm{A}$ is therefore a stable equilibrium and point $\mathrm{B}$ an unstable one. We have thus esthablished that a stable equilibrium with $\alpha<1$ must be such that the curve $\Gamma(x ; \lambda, \mu)$ is increasing, as in point $\mathrm{A}$. The following proposition summarises the case of partial channel substitution.

Proposition 5 (partial channel substitution) A stable equilibrium of the game with $\alpha<1$ has a share $\alpha$ of inexperienced consumers searching the conventional store and a share $1-\alpha$ searching the Internet, where $\alpha \in(0,1)$ is the solution to $\Gamma(x ; \lambda, \mu)=0$. Inexperienced consumers searching the Internet buy whenever 
$\min \left[p^{i}, p^{j}\right] \leq \lambda v$, that is, with probability $\pi$. Firms randomly select prices from the set $\left[\frac{x v}{2-x}, v\right]$ according to the cumulative distribution function given in (5).

\subsection{Characterization of Equilibria}

After having introduced the following three facts, we are ready to provide a complete characterization of stable equilibria in our model:

Fact $6 \frac{\partial \Gamma(x ; \lambda, \mu)}{\partial \lambda}>0$.

Fact $7 \Gamma(x ; 1 / 2, \mu)<0$.

Fact 8 For any $0<\mu<1, \Gamma(x ; 1, \mu)>0$ for $0<x<1$ and $\Gamma(1 ; 1, \mu)=\Gamma(0 ; 1, \mu)=$ 0

These facts tell us that as $\lambda$ increases, the incentives to search the Internet increase. This is easily understood if one considers the fact that $\lambda$ describes the confidence in the online purchase environment. Furthermore, when $\lambda \leq 1 / 2$ there is no incentive to visit the Internet, since the importance of a tangible purchase environment outweighs the importance of price comparison in that case, regardless of $\mu$ or $x$. When $\lambda=1$ there is no incentive to visit the store since the advantage of physical interaction is absent in that case.

The following statement is proved in the Appendix:

Theorem 6 There exists $\bar{\mu}$ such that

(A) for all $\mu>\bar{\mu}$, there exist a $\widetilde{\lambda}(\mu)$ such that the unique equilibrium is characterized by $\alpha=1$ for all $\lambda \leq \widetilde{\lambda}(\mu)$ and the unique equilibrium is characterized by $\alpha \in(0,1)$ for all $\lambda>\widetilde{\lambda}(\mu)$.

(B) for all $\mu \leq \bar{\mu}$, there exist $\widetilde{\lambda}(\mu)$ and $\widetilde{\widetilde{\lambda}}(\mu)$, with $\widetilde{\lambda}(\mu) \leq \widetilde{\widetilde{\lambda}}(\mu)$, such that

(i) the unique equilibrium is characterized by $\alpha=1$ for $\lambda \leq \tilde{\lambda}(\mu)$,

(ii) for $\widetilde{\lambda}(\mu)<\lambda<\widetilde{\widetilde{\lambda}}(\mu)$, there are two stable equilibria, one in which $\alpha=1$ and one in which $\alpha \in(0,1)$ and

(iii) for $\lambda>\widetilde{\widetilde{\lambda}}(\mu)$, the unique stable equilibrium is such that $\alpha \in(0,1)$.

The Theorem is illustrated in Figure (A1) for $\lambda \geq 1 / 2$, which shows simulation results obtained by using Microsoft Excel. Basically, there are three regions of parameters: one in which no channel substitution occurs, one in which partial channel substitution occurs and one region of multiple equilibria in which either partial or no substitution occurs. We know by Fact 7 that for all $\lambda<1 / 2$ the unique equilibrium is such that channel substitution does not occur. 


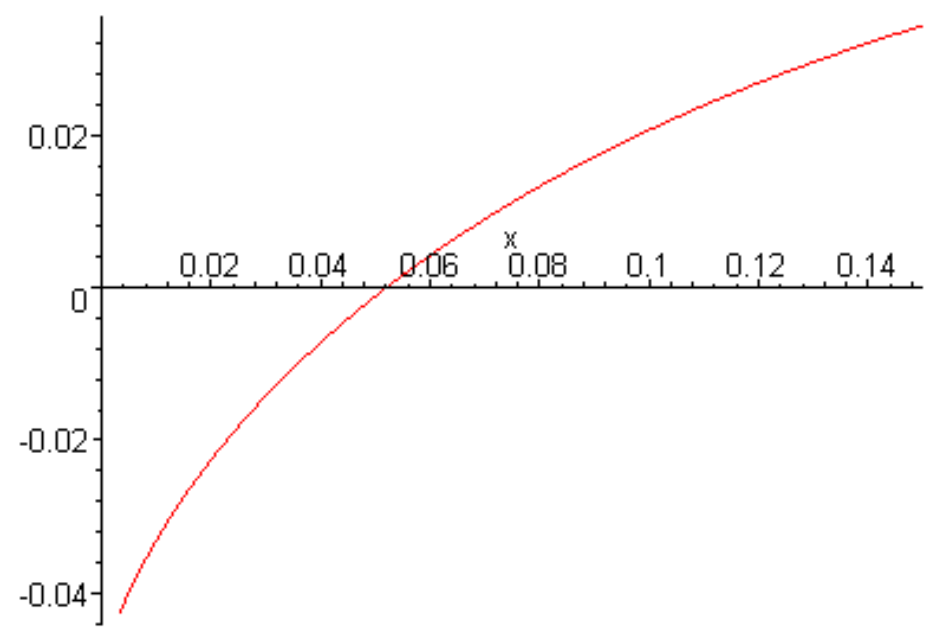

Figure $2: \Gamma(x ; 0.9, .93)$ for $x \in[0,1-\mu]$. Determination of equilibrium for a mature market.

\section{[Insert Figure A1 here]}

The two possibilities A and B introduced in the Theorem are illustrated in Figures (2) and (3), respectively. Figure (2) exhibits a mature market where $\mu=.85>\bar{\mu}$. In this case, the segment of the market on which firms exercise market power, $x \equiv \alpha(1-\mu)$, is necessarily small. Therefore $\Gamma(x ; \lambda, \mu)$ is only increasing; $x$ cannot become large enough to allow monopolistic pricing to diminish price dispersion in the market. In this case the equilibrium is unique; depending on $\lambda$ either no channel substitution occurs or partial channel substitution occurs. In the picture, an equilibrium with partial channel substitution is found where the curve intersects the horizontal axis.

On the other hand, for an immature market $(\mu=0.3), x$ may become large and then the shape is as in Figure (3). Depending on the degree of online uncertainty $\lambda$, there may be one or two equilibria: if $\lambda$ is low enough, $\Gamma(x ; \lambda, \mu)<0$ for all $\alpha$ and then the only equilibrium is $\alpha=1$, hence no channel substitution occurs. For higher $\lambda$, we obtain one equilibrium with $\alpha \in(0,1)$ where $\Gamma(x ; \lambda, \mu)=0$ and one with $\alpha=1$ where $\Gamma(1-\mu ; \lambda, \mu)<0$. Finally, for $\lambda$ high enough, the unique equilibrium has $\alpha \in(0,1)$.

The properties of the equilibria will be discussed in detail below. At this point we already would like to mention the following observations to which the Theorem points. Firstly, for all degrees of market maturity, if the online inconvenience is sufficiently high (low $\lambda$ ), and hence physical aspects are important, inexperienced consumers will not substitute the online for the offline channel. Secondly, for relatively immature markets, another reason for channel substitution not to occur is the following. In an 


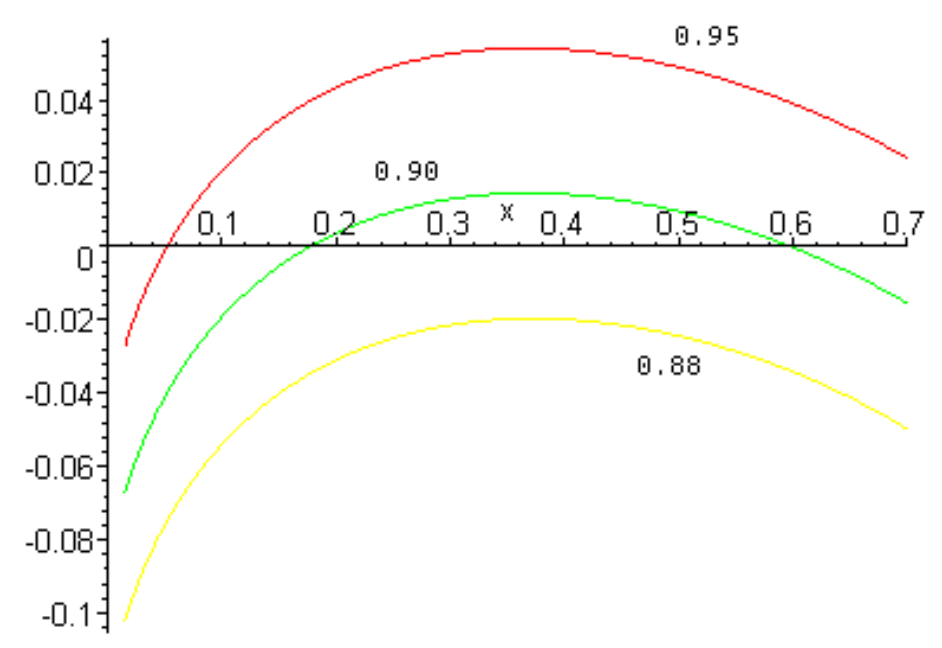

Figure 3: $\Gamma(x ; \lambda, 0.3)$ in $x \in[0,1-\mu]$ for different levels of $\lambda$. Illustration of equilibrium for an immature market.

immature market, the decisions of inexperienced consumers are important for pricing behavior. If all consumers coordinate on using the physical store, this shopping behavior induces monopolistic pricing, and hence, consumers infer that they cannot make a purchase on the electronic retail channel (since $\pi=0$ ) and an equilibrium of no channel substitution may prevail.

\section{Comparisons across Markets}

We will now discuss our analysis of the issues raised in the Introduction. Specifically, we will analyze the market sizes of the two channels, the total market size and the expected price resulting from market interaction. We will argue that if we specify online inconvenience as the inability to inspect a product, the parameter $\lambda$ describes a product market.

\subsection{Retail Channel substitution}

In this subsection we address the question how market circumstances affect the extent to which consumers will substitute the online for the offline retail channel. In doing so, we gain insight in the functioning of electronic markets. Furthermore, we will explain empirical differences in online penetration across markets.

To determine the relative importance of the electronic retail channel we have to look at the decisions by the $(1-\mu)$ inexperienced consumers, since the experienced consumers are already conquered by the electronic channel. A share $\alpha$ of them frequents the store, therefore the relative size of the conventional and the electronic 
channel is $x$ and $(1-x)$, respectively. As we have shown in Section 3 , the variable $x$ is determined by two factors. The valuation parameter $\lambda$, reflecting the confidence in an online purchase environment, and the maturity of the market, $\mu$. We will discuss the impact of both determinants in turn, in each case distinguishing between partial and no channel substitution.

\section{Online uncertainty: $\lambda$.}

The greater the uncertainty or inconvenience online, the more consumers are tempted to use the conventional store. Indeed, we have already demonstrated in Theorem 6 that all inexperienced consumers will use the store for $\lambda$ sufficiently low, that is, $\lambda \leq \widetilde{\lambda}(\mu)$. For these levels of $\lambda$ there occurs no channel substitution and then the market share of the online channel is simply given by $1-x \equiv \mu$. For $\lambda>\widetilde{\lambda}(\mu)$ partial channel substitution may prevail, with a fraction of inexperienced consumers using the online channel. We expect that as $\lambda$ increases, this fraction increases. To investigate this hypothesis, we can use Fact 6 introduced above and the fact that in a stable equilibrium, $\frac{\partial \Gamma(\cdot)}{\partial x}>0$ combined with the Implicit Function Theorem to obtain the result $\frac{\partial x}{\partial \lambda}<0$ if $\alpha<1 .{ }^{12}$ Thus if channel substitution is partial and $\lambda$ decreases, $\alpha$ increases. This result supports our hypothesis: as $\lambda$ decreases less buyers frequent the online retail channel.

If we interpret the online inconvenience as resulting from the inability to physically inspect a product before purchase, we can relate the value of $\lambda$ to the degree of nondigital product variety: the higher the non-digital variety, the more important it is to inspect non-digital product attributes, hence, the lower $\lambda$.

In this framework, we can infer from our analysis that product markets which are characterised by a high degree of non-digital product variety, are likely to have relatively small electronic retail channels. Vice versa, we expect more intensive use of the electronic channel for products that show little non-digital variety. We illustrate this result in Figure (A2), where the sales in the conventional channel are depicted.

\section{[Insert Figure A2 here]}

Our characterization is supported empirically. The electronic channel makes up a small share of retailing in product categories as clothing, food, flowers, cards, home/gardening supplies and gifts (0.1\% or less in the US and Europe), while online penetration is biggest for categories such as computer hardware/software and books (in the US respectively $9.2 \%$ and $5.1 \%$, in Europe these figures are $3.5 \%$ and $1.6 \%$ resp.). ${ }^{13}$ We may note that these figures show an enormous gap in the extent of online penetration for the different product categories. These observations are in line with our predictions, specifically, one of our results is that there occurs a substantial

\footnotetext{
${ }^{12}$ The Implicit Function Theorem implies in the case under consideration that $\frac{d x}{d \lambda}=-\frac{\partial \Gamma(\cdot) / \partial \lambda}{\partial \Gamma(\cdot) / \partial x}<0$.

${ }^{13}$ The observations concern the year 1999, Boston Consulting Group 2000.
} 
jump in market shares, when consumers switch to partial channel substitution, see Figure (A2). Furthermore, since in the United States electronic commerce is more widely developed, it is convincing to observe that even there the online penetration for the highly differentiated categories is very low compared to the less differentiated categories.

\section{The maturity of the market: $\mu$.}

The maturity of the market influences the expected price difference and therefore the best-price uncertainty for offline shoppers. We demonstrate in the Appendix that

Fact $9 \frac{\partial x}{\partial \mu}>0$ if $\alpha<1$.

Clearly, there are two effects of $\mu$ at work. Recall that $x \equiv \alpha(1-\mu)$ so that the direct effect of an increase in $\mu$ is to decrease $x$. However, there is also an indirect effect through the impact on $\alpha$. This indirect effect is positive as an increase in $\mu$ increases $\alpha$. This can be understood by taking into account that the more experienced consumers there are in the market, more price comparisons are being made, and the less interested inexperienced consumers are in making price comparisons themselves. Surprisingly, the latter indirect effect dominates the former! Therefore, when a market matures, inexperienced consumers infer that the increased magnitude of experienced consumers reduces the price dispersion in the market. Hence, they frequent the Internet less often. This effect dominates the fact that there are less inexperienced consumers in total and $x$ increases. This process continues untill $\alpha$ becomes one, when only the first effect remains. In Figure (A3) we illustrate this effect.

[Insert Figure A3]

The figure shows that even though we can show that the impact of $\mu$ on $x$ is positive when $\alpha<1$, the impact is quite small. The figure also shows that when $\mu$ is small, there exist two equilibria. The important lesson here is that the presence of relatively many experienced consumers does not imply that expected prices are relatively low.

\subsection{Size of the market}

In the Introduction it was pointed out that some consumers who consider to buy online actually do not make a purchase. This phenomenon of drop-out must therefore be included in a realistic model of electronic commerce. Above we illustrated that the online consumers do not necessarily make a purchase. In Section 3.2 we showed that the $(1-\alpha)(1-\mu)$ inexperienced consumers who search the Internet only make 
the purchase if $\min \left[p^{i}, p^{j}\right] \leq \lambda v$. Therefore, the total number of transactions in the market is given by

$$
S(x ; \lambda, \mu) \equiv 1-(1-\pi)(1-\alpha)(1-\mu) .
$$

Below we will investigate the role the two market characteristics of our model play in determining market size $S$.

\section{Online uncertainty: $\lambda$.}

We first focus on the role of the parameter $\lambda$. A few observations can be made. First, when $\lambda$ is small enough, all inexperienced consumers visit the store $(\alpha=1)$ and there is no drop-out, i.e. $S(\cdot)=1$. Second, when $\lambda=1$ everybody visits the Internet $(\alpha=0)$, so that in equilibrium prices drop to zero and there is, again, no drop-out. In between, when $0<\alpha<1$, there will always be some drop-out as firms also randomize over prices larger than $\lambda v$. Hence, there is a non-monotonic relation between $S$ and $\lambda$. The reason for this non-monotonicity can be explained by looking at the expression for $S(x ; \lambda, \mu)$ and realizing that there are two forces at work. When $\lambda$ increases, the number of inexperienced consumers visiting the Internet increases, increasing the number of consumers that are prone to the drop-out phenomenon. However, when more consumers visit the Internet, the more competitive the firms' pricing behavior, thereby decreasing the chance that individual consumers drop out.

Our model thus predicts that the size of the market depends on the importance of physical interaction: for $\lambda$ large, physical interaction plays an insignificant role in the consumer purchase. As a consequence, many consumers use the Internet, leading to competitive prices. In turn, competitive pricing ensures that almost all inexperienced consumers indeed make the purchase, so that the entire demand side is active in the market. As $\lambda$ decreases, some consumers will prefer to search the conventional store in order to avoid online inconveniences. These consumers do not compare prices and therefore induce firms to price more monopolistically. In this way the consumers in the store impose a negative externality on the consumers searching the Internet: consumers comparing prices on the Net will more often drop-out. Because of this reason, the size of the market starts to decline. As $\lambda$ becomes small enough, all inexperienced consumers prefer to searche the store, and therefore all consumers make the purchase: the marketsize returns to its higher, initial level.

\section{The impact of Market Maturity $\mu$.}

It is easily checked that $\frac{\partial S(x ; \lambda, \mu)}{\partial \mu}=(1-\pi) \frac{\partial x}{\partial \mu}+(1-\alpha)(1-\mu) \frac{\partial \pi}{\partial \mu}+(1-\pi)$. When $\alpha=1$ we have that $\frac{\partial x}{\partial \mu}=-1$ and hence, $\frac{\partial S(\cdot)}{\partial \mu}=0$. For the case of $\alpha<1$ we will consider each term. Firstly, experienced consumers always buy, and hence, there are more transactions in the market if there are more consumers of this type. This is reflected by the third term. Secondly, the second term shows us that the inexperienced 
online shoppers will more often make a purchase, since $\frac{\partial \pi}{\partial \mu}>0$, and thirdly, the first term tells us that more inexperienced consumers will use the store, that is, the channel where they will make the purchase for sure. Therefore, the three terms are positive and the number of transactions increases in market maturity when channel substitution is partial.

\subsection{Prices}

We finally investigate the impact of market circumstances on price setting behavior. Since our model exhibits price dispersion, we will look at the expected price. In Lemma 4 we have shown that the expected price equals:

$$
E(p)=\frac{x v}{2(1-x)} \ln \left[\frac{\lambda(2-x)}{x}\right]+\frac{x v}{2 \mu} \ln \left[1+\frac{(1-\lambda) \mu}{\lambda(1-x)}\right] .
$$

As before, we will investigate the impact of market determinants $\lambda$ and $\mu$.

\section{Online uncertainty: $\lambda$}

In the appendix, we establish the following property:

Fact $10 \frac{d E(p)}{d \lambda}<0$ if $\alpha<1$ and $\frac{d E(p)}{d \lambda}=0$ if $\alpha=1$

Fact 10 tells us that the expected price is nondecreasing in the importance of tangible interaction. This result can be understood by considering that if $\alpha<1$, $\frac{d E(p)}{d \lambda}=\frac{\partial E(p)}{\partial x} \frac{\partial x}{\partial \lambda}+\frac{\partial E(p)}{\partial \lambda}<0$. The first term tells us that as $\lambda$ increases, less consumers use the store and therefore, firms price more competitively, hence, this term is negative. The second term, $\frac{\partial E(p)}{\partial \lambda}$, is also negative, illustrating that an increase in $\lambda$ causes $\pi$ to increase, thereby making it more beneficial to have the best price in the market as it attracts more purchases. See also Figure (A2) and Table (T1).

\section{Market maturity $\mu$}

For an equilibrium of no channel substitution, we can easily infer from (3) that $\frac{\partial F(p)}{\partial \mu}>0$, hence the expected price decreases in market maturity in such an equilibrium. If channel substitution is partial, we obtain that $\frac{d E(p)}{d \mu}=\frac{\partial E(p)}{\partial x} \frac{\partial x}{\partial \mu}+\frac{\partial E(p)}{\partial \mu}$ and from Fact 9 and the proof of Fact 10 we know that the first, indirect, effect is positive. We can also show that the direct effect is negative. However, by simulation we can obtain that the direct effect is offset by the positive indirect one (see Table (T1)). This overall effect is small, but positive, which is a quite remarkable result: an increase in the proportion of price comparing shoppers leads to an increase in expected price! The intuition is the same as for Fact 9: an increase in $\mu$ causes more inexperienced consumers to use the store, such that the total number of consumers in the store, $x$, rises. For higher $x$ firms are induced to set higher prices. 
[Insert Table T1]

\section{Discussion and Conclusion}

In this paper we have set up a model of electronic commerce, describing how consumers choose between offline and online retail channels. Our analysis shows how the degree of retail channel substitution is determined, by considering the consumer's trade off between price comparisons and a tangible, immediate transaction.

We took the need to physically compare different versions of a product as the leading example of an online inconvenience. In this interpretation, we demonstrated that for a particular product market, the sales in each channel depend on the importance of physical inspection and on the number of consumers already familiar with the product. Our main results are as follows. Firstly, we expect online penetration to be low for the product categories that are characterised by the presence of non-digital product variety. More specifically, for product variety high enough, the consumers that need to learn product attributes do not use the online channel at all and for lower levels of product variety, the share of online sales is decreasing in product variety. Secondly, we found that online consumers do not always buy because of the reason that their uncertainty about the product match is not always compensated by a low enough observed price. Thirdly, we investigated the pricing behavior of firms and we found that firms price above the competitive level because they have market power over the consumers in their store. Additionally, the tension between having monopoly power in the store and competition on the Internet yields price dispersion in the market. The expected price in the market is increasing in the importance of the online uncertainty if some inexperienced use the online channel and constant if no inexperienced consumer uses the online channel.

As was mentioned in the Introduction, other reasons than physical inspection may give rise to the perception of online uncertainty. Examples are the payment method or the slow delivery of goods. In these cases, the parameter $\lambda$ could describe the infrastructural and institutional state of affairs of Internet transactions or cultural determined attitudes towards electronic commerce. In this wider interpretation our results can be described as follows: If the perceived inconvenience is large enough, electronic commerce will only be used by the users in the economy who do not incur it. If some inexperienced consumers do shop online, the market share of the Internet is decreasing in the inconvenience.

All the above results are in line with observations that have been made on Internet purchases. We also found two quite unexpected results, namely that the market share of the electronic channel is decreasing and the expected price is increasing in the proportion of price comparing experienced consumers in the market. Finally, the fact 
that the expected price may rise in the online uncertainty is somewhat surprising at first sight: one would expect that firms would try to compensate the consumer for uncertainties, by lower prices. However, a firm rather prefers the consumer to feel uncertain indeed, and to shop where she is not exposed to the rival's offers.

In the remainder of this section we discuss the importance of two main features of our model: firms set the same price on both retail channels and consumers search only once.

Concerning the pricing policy, there are different ways to defend this assumption. Firstly, one may think that a firm will be punished by consumers if they find out that a firm discriminates between the two channels. ${ }^{14}$ Secondly, one could also argue that the presence of administrative menu cost of having two prices prohibits firms to price discriminate between retail channels. Finally, suppose that firms choose two different prices, one for each channel. Then the equilibrium is such that firms charge $p=0$ on the Internet, $p=\lambda v$ in the store and all inexperienced consumers would search the store. For a set of values of $\lambda$ and $\mu$, firms obtain lower expected profits, however, than in the case we studied. One may therefore expect to find a Prisoner's Dilemma type of problem: both firms are better off if both pursue equal pricing than if both differentiate, but they individually have an incentive to differentiate. The reason for this is that equal pricing limits price competition on the electronic channel, which is in the interest of both firms. In a repeated setting, there exist then 'cooperative' type of equilibria where firms concentrate on the strategies we have considered in the main body of the paper.

The framework that we have studied has the consumer searching only once. However, it seems likely that consumers are tempted to use both channels for their purchase. They might for instance first select the firm with the best price by browsing the Internet, and subsequently visit the store of that firm to inspect the product line and make the purchase. Similarly, a consumer that has evaluated products in a particular store might decide to buy the preferred product version online, at the best price. In the remainder we discuss how our model could be extended to allow for more than one search, again taking product match uncertainty as an example of an online inconvenience. We will argue that our qualitative results will hold in such an extension.

Multiple search can be accounted for by allowing consumers to search sequentially, introducing different search $\operatorname{costs} c_{s}$ and $c_{I}$ for the store and the Internet, where $0<c_{I} \leq c_{s}$. Consumers first decide which channel to search. After having observed what there is to be observed (prices $\left(p^{1}, p^{2}\right)$ of different stores on the Internet, all

\footnotetext{
${ }^{14}$ One could oppose to this argument by saying that consumers understand that the two channels differ in their transaction costs, both on the demand and supply side, and would therefore accept a price differential. However, we do not consider transaction costs on the demand side and we think consumers do not have that much consideration towards the efficiency of the firm.
} 
product characteristics and one price in the store), consumers may decide to enter a second stage by engaging in an additional search. Therefore, if search costs are not prohibitive, the inexperienced consumer may find it optimal to eliminate both bestprice uncertainty as well as product match uncertainty, by performing more than one search. If this were the case, however, all consumers would make price comparisons and Bertrand prices would result. Clearly, when $(1-\lambda) v>\left(c_{s}-c_{I}\right)$, this cannot be an equilibrium, as argued in Lemma 1: since the expected price difference is zero, consumers prefer to visit the store and buy the product that best matches their preferences (even it may be somewhat more costly in terms of search time) so that they will have no reason to search the Internet.

To give a sketch of possible equilibrium configurations, consider the following four strategies of the inexperienced consumer: (i) "go to the Internet and buy from the cheapest site", (ii) "go to one of the stores and buy the product that you like best", (iii) "go to the Internet first and afterwards go to the store that has the lowest price and buy the product you like best in the store" and, finally, (iv) "go to one of the stores and buy the product that you like best if the price is below a certain upperbound, otherwise continue searching on the Net and buy from the lowest priced site". If the consumer can make a purchase on the Internet, then strategy (i) and (iii) have a clear dominance relation to each other, namely, if $c_{s}>(1-\lambda) v$, then strategy (i) dominates strategy (iii). If the reverse inequality holds, then strategy (iii) dominates strategy (i). This easily follows from the fact that the cost and expected benefits of an additional search in the conventional channel are $c_{s}$ and $(1-\lambda) v$, respectively. On the other hand, a version of strategy (iv), namely "go to one of the stores and buy the product that you like best if the price is smaller than $E(p)+c_{I}$, otherwise continue searching on the Net and buy from the lowest priced site" clearly dominates strategy (ii) and other strategies in class (iv). This is easily seen from the fact that the expected price the other firm charges equals $E(p)$ and one first has to incur the search $\operatorname{cost} c_{I}$.

Given these observations on the optimal search strategy of the consumers, it follows that inexperienced consumers will either randomize over strategy (i) and the ' $E(p)+c_{I}$ ' version of (iv), namely if $c_{s}>(1-\lambda) v$, or randomize over this last strategy and strategy (iii). It is also clear that some inexperienced consumers will not choose strategy (i) or (iii) for a reason similar to the one argued in Lemma 1. Moreover, in equilibrium the upper bound of the price distribution equals $\min \left[E(p)+c_{I}, v\right]$ as a firm who charges the maximal price will only attract costumers who visit the conventional store and if he would charge a price above $E(p)+c_{I}$ he will also loose these costumers. Hence, given this reaction by the firm to the threat to continue searching on the Net, in equilibrium no consumer will first visit the store to inquire which product fits his tastes best and then go and look for the lowest price on the Internet. So, even if we allow for sequential search, firms have some market power 
(bounded above by the search cost) and some inexperienced consumers will not use the sequential search possibility, but simply buy at the conventional store. Most qualitative results of the paper will therefore most likely continue to hold, although the analysis will become more complicated.

\section{Appendix}

\section{Proof of Facts}

The equilibrium condition for $\alpha \in(0,1)$ and $0 \leq x \leq \bar{x}$ is given by:

$$
\begin{aligned}
\Gamma(x ; \lambda, \mu)=\frac{x}{(1-x)^{2}}\left(\frac{x}{4}\left(4-\lambda+\frac{1}{\lambda}\right)-1\right) & +\frac{x}{2(1-x)^{2}} \ln \frac{\lambda(2-x)}{x}+ \\
& +\frac{x}{2 \mu} \ln \left[1+\frac{(1-\lambda) \mu}{\lambda(1-x)}\right]-1+\lambda=0 .
\end{aligned}
$$

Fact 1: $\Gamma(0 ; \lambda, \mu)<0$ for all $\lambda<1$ and all $\mu ; \Gamma(0 ; 1, \mu)=0$ for all $\mu$.

Proof of Fact 1: It is easy to see that $\Gamma(0 ; \lambda, \mu)=-1+\lambda$, the result then follows.

Fact 2: $\Gamma(\bar{x} ; \lambda, \mu)<0$ for all $\mu>0$ and all $\lambda<1 ; \Gamma(\bar{x} ; \lambda, 0)=0$ for all $\lambda$.

Proof of Fact 2: It is easy to see that

$\Gamma(\bar{x} ; \lambda, \mu)=-1+\frac{\lambda}{\mu(\lambda+1)} \ln \left[1+\frac{\mu(1+\lambda)}{\lambda}\right]$. Now substituting $z=\frac{\mu(\lambda+1)}{\lambda}$, we have to show that

$$
-1+\frac{\ln (1+z)}{z} \leq 0
$$

For the case $\mu>0$ we have that $z>0$. As $\frac{\ln (1+z)}{z}<1$ for all $z>0, \Gamma(\bar{x} ; \lambda, \mu)<0$ for $\mu>0$. For the case $\mu=0$ it is enough to observe that $\lim _{z \rightarrow 0} \frac{\ln (1+z)}{z}=1$.

Fact 3: $\left.\frac{\partial \Gamma(x ; \lambda, \mu)}{\partial x}\right|_{x=0}>0$.

Proof of Fact 3: This is easily verified by first substituting $\mu=1$ and taking the derivative:

$$
\begin{aligned}
& \frac{\partial \Gamma(x ; \lambda, 1)}{\partial x}=\frac{x}{2 \lambda(1-x)^{3}}\left(2 \lambda+2-(1-\lambda)^{2}\right)-\frac{1+x}{(1-x)^{3}}+ \\
& \quad+\frac{1+x}{2(1-x)^{3}} \ln \frac{\lambda(2-x)}{x}-\frac{1}{(2-x)(1-x)^{2}}+ \\
& \quad+\frac{1}{2} \ln \left[1+\frac{(1-\lambda)}{\lambda(1-x)}\right]+\frac{x}{2}\left[\frac{(1-\lambda)}{\lambda(1-x)^{2}+(1-\lambda)(1-x)}\right]
\end{aligned}
$$


Evaluating this expression at $x=0$ gives $+\infty$. If we then take into account that $\frac{\Gamma^{2}(x ; \lambda, \mu)}{\partial x \partial \mu}<0$, shown in observation (ii), step 2 of Fact 4 , it easily follows that the claim holds for all $\mu$.

Fact 4: There exist at most two values of $x \in[0, \bar{x}]$ such that $\Gamma(x ; \lambda, \mu)=0$.

Proof of Fact 4: We proceed in 2 steps; first we concentrate on $\mu=0$. The second step is to establish that the claim holds also for $\mu>0$.

1 For $\mu=0$ the equilibrium condition is

$$
\begin{aligned}
\Gamma(x ; \lambda, 0)=\frac{x^{2}\left(4 \lambda+1-\lambda^{2}\right)}{4 \lambda(1-x)^{2}}- & \frac{x}{(1-x)^{2}}-1+\lambda+ \\
& +\frac{x}{2(1-x)^{2}} \ln \frac{\lambda(2-x)}{x}+\frac{(1-\lambda) x}{2 \lambda(1-x)}=0 .
\end{aligned}
$$

We have that

$$
\begin{aligned}
\frac{\partial \Gamma(x ; \lambda, 0)}{\partial x}=\frac{x\left(4 \lambda+1-\lambda^{2}\right)}{2 \lambda(1-x)^{3}}-\frac{1+x}{(1-x)^{3}}+ \\
\quad+\frac{1+x}{2(1-x)^{3}} \ln \frac{\lambda(2-x)}{x}-\frac{1}{(2-x)(1-x)^{2}}+\frac{(1-\lambda)}{2 \lambda(1-x)^{2}}
\end{aligned}
$$

and

$$
\begin{aligned}
& \quad \frac{\partial^{2} \Gamma(x ; \lambda, 0)}{\partial x^{2}}=\frac{(1+2 x)\left(4 \lambda+1-\lambda^{2}\right)}{2 \lambda(1-x)^{4}}-\frac{4+2 x}{(1-x)^{4}}+ \\
& +\frac{2+x}{(1-x)^{4}} \ln \frac{\lambda(2-x)}{x}+\frac{5-3 x}{(2-x)^{2}(1-x)^{3}}-\frac{1+x}{x(2-x)(1-x)^{3}}+\frac{(1-\lambda)}{\lambda(1-x)^{3}} .
\end{aligned}
$$

Furthermore, $\frac{\partial^{2} \Gamma(x ; \lambda, 0)}{\partial x^{2}}>0$ iff $g(x, \lambda) \equiv \frac{2+x}{1-x} \frac{\left(1-\lambda^{2}\right)}{2 \lambda}-2+\frac{2+x}{(1-x)} \ln \frac{\lambda(2-x)}{x}-\frac{2(1-x)^{2}}{x(2-x)^{2}}>$ 0.

Now the following observations (i) to (iii) establish that there exists exactly one $\widehat{x}$ such that $\left.\frac{\partial^{2} \Gamma(x ; \lambda, 0)}{\partial x^{2}}\right|_{x=\widehat{x}}=0$. This fact, in combination with Facts 1 and 2 ensures that the curve $\Gamma(x ; \lambda, 0)$ intersects the horizontal axis at most twice on the relevant interval $\left[0, \frac{2(1+\lambda)}{3+\lambda}\right]$. (Note that, moreover, if there are two intersection points, $\partial^{2} \Gamma(\cdot) / \partial x^{2}<0$ whenever $\partial \Gamma(\cdot) / \partial x>0$ as otherwise $\Gamma(\cdot)$ will be only increasing).

(i) $g(0, \lambda)<0$.

We obtain $\lim _{x \rightarrow 0} g(x, \lambda)=\frac{\left(1-\lambda^{2}\right)}{\lambda}-2+\lim _{x \rightarrow 0}\left\{\frac{2+x}{(1-x)} \ln \frac{\lambda(2-x)}{x}-\frac{2(1-x)^{2}}{x(2-x)^{2}}\right\}$. Now it is enough to observe that the second term decreases faster to $-\infty$ than the first increases to $+\infty$. 
(ii) $g(\bar{x}, \lambda)>0$.

We have that: $g(\bar{x}, \lambda)=\frac{8 \lambda^{2}+4 \lambda+4-(1-\lambda)\left(1-\lambda^{2}\right)}{4 \lambda}>0$.

(iii) There exists at most one $\widehat{\widehat{x}}$ such that $\left.\frac{\partial g(x, \lambda)}{\partial x}\right|_{x=\widehat{\widehat{x}}}=0$.

We proceed as follows. We construct a function that has the sign of $\frac{\partial g(x, \lambda)}{\partial x}$ and then we show that this function is monotone. We have:

$$
\begin{aligned}
& \frac{\partial g(x, \lambda)}{\partial x}=\frac{3\left(1-\lambda^{2}\right)}{2 \lambda(1-x)^{2}}+\frac{3}{(1-x)^{2}} \ln \frac{\lambda(2-x)}{x}+\frac{2(1-x)\left(2-x+x^{2}\right)}{x^{2}(2-x)^{3}}-\frac{2(2+x)}{(1-x) x(2-x)}, \text { so that } \\
& \frac{\partial g(x, \lambda)}{\partial x}>0 \text { iff } \\
& h(x, \lambda) \equiv \frac{3\left(1-\lambda^{2}\right)}{2 \lambda}+3 \ln \frac{\lambda(2-x)}{x}+\frac{2(1-x)^{3}\left(2-x+x^{2}\right)}{x^{2}(2-x)^{3}}-\frac{2(2+x)(1-x)}{x(2-x)}>0 .
\end{aligned}
$$

Now we will show that $\frac{\partial h(x, \lambda)}{\partial x}<0$. Consider:

$$
\begin{aligned}
& \frac{\partial h(x, \lambda)}{\partial x}=\frac{-6}{x(2-x)}+\frac{2(1-x)^{2}}{x^{3}(2-x)^{4}}\left[\left(-5 x^{2}+6 x-7\right) x(2-x)-(4-5 x)(1-x)\left(2-x+x^{2}\right)\right]+ \\
& \frac{2+4 x}{x(2-x)}+\frac{4(2+x)(1-x)^{2}}{x^{2}(2-x)^{2}}=\frac{-4(1-x)}{x(2-x)}+\frac{2(1-x)^{2}}{x^{2}(2-x)^{2}}\left[\frac{-5 x^{3}+x^{2}-4+2 x}{x(2-x)}+2(2+x)\right]-\frac{2(4-5 x)(1-x)^{3}}{x(2-x)^{4}}(\mathrm{~A} 1)
\end{aligned}
$$

We can show that (A1) is negative. However, the proof is somewhat messy, but straightforward. It is available on request. Therefore, the proof of observations (i) to (iii) is now complete. They show that $\Gamma(x ; \lambda, 0)$ intersects the horizontal axis at most twice.

$2 \mu>0$.

In step 1 we have shown that $\Gamma(x ; \lambda, 0)=0$ occurs for at most two values of $x$. Now we will show that the claim holds for all $\mu$. We prove this by considering partial derivatives of $\Gamma(\cdot)$ with respect to $\mu$, for fixed $x$. The argument, based on the following three observations, will be discussed below.

(i) Keeping $x$ fixed, $\frac{\partial \Gamma(x ; \lambda, \mu)}{\partial \mu}<0$.

We have that $\frac{\partial \Gamma(x ; \lambda, \mu)}{\partial \mu}=-\frac{x}{2 \mu^{2}} \ln \left[1+\frac{(1-\lambda) \mu}{\lambda(1-x)}\right]+\frac{x}{2 \mu} \frac{(1-\lambda)}{\lambda(1-x)+(1-\lambda) \mu}<0$ if

$\ln \left[1+\frac{(1-\lambda) \mu}{\lambda(1-x)}\right]>\frac{(1-\lambda) \mu}{\lambda(1-x)+(1-\lambda) \mu}$.

Note that $L H S(\mu=0)=R H S(\mu=0)$ and that

$\frac{\partial L H S}{\partial \mu}=\frac{1-\lambda}{\lambda(1-x)+(1-\lambda) \mu}, \frac{\partial R H S}{\partial \mu}=\frac{(1-\lambda)[\lambda(1-x)+(1-\lambda) \mu]-(1-\lambda)^{2} \mu}{[\lambda(1-x)+(1-\lambda) \mu]^{2}}$ and thus $\frac{\partial L H S}{\partial \mu}>\frac{\partial R H S}{\partial \mu}$. Therefore, $\frac{\partial \Gamma(x ; \lambda, \mu)}{\partial \mu}<0$.

(ii) The second derivative $\frac{\partial^{2} \Gamma(x ; \lambda, \mu)}{\partial x \partial \mu}<0$.

We obtained under (i) that $\frac{\partial \Gamma(x ; \lambda, \mu)}{\partial \mu}=-\frac{x}{2 \mu^{2}} \ln \left[1+\frac{(1-\lambda) \mu}{\lambda(1-x)}\right]+\frac{x}{2 \mu} \frac{1-\lambda}{\lambda(1-x)+(1-\lambda) \mu}$. Taking the derivative with respect to $x$ we have

$2 \mu \frac{\partial^{2} \Gamma(x ; \lambda, \mu)}{\partial \mu \partial x}=-\frac{1}{\mu} \ln \left[1+\frac{(1-\lambda) \mu}{\lambda(1-x)}\right]-\frac{x(1-\lambda)}{\lambda(1-x)^{2}+(1-\lambda)(1-x) \mu}+\frac{1-\lambda}{\lambda(1-x)+(1-\lambda) \mu}+\frac{x(1-\lambda) \lambda}{[\lambda(1-x)+(1-\lambda) \mu]^{2}}$.

Under (i) we have shown that the first and third term together are negative.

Therefore we only consider the second and fourth terms. Rewriting yields

$\frac{-x(1-\lambda)}{\lambda(1-x)+(1-\lambda) \mu}\left[\frac{1}{1-x}+\frac{\lambda}{\lambda(1-x)+(1-\lambda) \mu}\right]$, which is obviously negative. 
(iii) The third derivative, $\frac{\partial^{3} \Gamma(x ; \lambda, \mu)}{\partial x^{2} \partial \mu}<0$.

It is convenient to consider the term of $\Gamma(\cdot)$ that depends on $\mu$ and then take the second derivative to $x$. Then we obtain the following: $A \equiv\left[\frac{(1-\lambda)}{(1-x)^{2} \lambda+(1-\lambda)(1-x) \mu}\right]+$ $\frac{x(1-\lambda)[2 \lambda(1-x)+(1-\lambda) \mu]}{2\left[(1-x)^{2} \lambda+(1-\lambda)(1-x) \mu\right]^{2}}$. The first term is decreasing in $\mu$, therefore it suffices to demonstrate that the second term is decreasing in $\mu$. The second term can be rewritten as $\frac{x(1-\lambda)}{(1-x)}\left[2-\frac{(1-\lambda) \mu}{(1-x)(\lambda)+(1-\lambda) \mu}\right]$ and the sign of the derivative with respect to $\mu$ is the sign of $\frac{-(1-\lambda) \lambda(1-x)}{[\lambda(1-x)+(1-\lambda) \mu]^{2}}$, which is clearly negative.

We will now describe how the above three facts can be used to complete the argument for $\mu>0$. Firstly, on the domain where $\Gamma(x ; \lambda, 0)<0$ we have $\Gamma(x ; \lambda, \mu)<$ 0 because of observation (i). Hence, no intersections arise. Secondly, on the domain where $\Gamma(x ; \lambda, 0)$ is decreasing, $\Gamma(x ; \lambda, \mu)$ is decreasing as well because of observation (ii). Therefore on this domain there can emerge at most one intersection. Thirdly, on the domain where $\Gamma(x ; \lambda, 0) \geq 0$ and increasing, we have that $\frac{\partial^{2} \Gamma(x ; \lambda, 0)}{\partial x^{2}}<0$. Then because of observation (iii) given in step 2 above it must be that $\frac{\partial^{2} \Gamma(x ; \lambda, \mu)}{\partial x^{2}}<0$ as well on this domain. This concavity property implies that at most two intersections arise and if two intersections arise, there does not arise any intersection on the domain mentioned secondly.

Therefore, $\Gamma(x ; \lambda, \mu)=0$ occurs for at most two values of $x$.

Fact 5: $\left.\frac{\partial^{2} \Gamma(x ; \lambda, \mu)}{\partial x^{2}}\right|_{x=0}<0$.

Proof of Fact 5: This property is established for $\mu=0$ in the Proof of Fact 4 , step 1, observation (i). Now since we demonstrated in step 2 of that proof that $\frac{\Gamma^{3}(x ; \lambda, \mu)}{\partial x^{2} \partial \mu}<0$ the claim holds for all $\mu$.

Fact 6: $\frac{\partial \Gamma(x ; \lambda, \mu)}{\partial \lambda}>0$.

Proof of Fact 6. We can compute that

$$
\frac{\partial \Gamma(\cdot)}{\partial \lambda}=\frac{x}{(1-x)^{2}}\left[\frac{2 \lambda-x \lambda^{2}-x}{4 \lambda^{2}}\right]+\frac{x(1-x-\mu)}{2 \mu[\lambda(1-x)+(1-\lambda) \mu]}+\frac{\mu 2 \lambda-x}{\mu 2 \lambda} .
$$

This is larger than zero if

$$
k(x ; \lambda, \mu) \equiv \frac{1}{(1-x)^{2}}\left[\frac{2 \lambda-x \lambda^{2}-x}{4 \lambda^{2}}\right]+\frac{(1-x-\mu)}{2 \mu[\lambda(1-x)+(1-\lambda) \mu]}+\frac{1}{x}-\frac{1}{2 \mu \lambda}>0 .
$$

We can show that, for fixed $x$ :

$$
\frac{\partial k(\cdot)}{\partial \mu}=\frac{-2 \mu[\lambda(1-x)+(1-\lambda) \mu]-(1-x-\mu)[2 \lambda(1-x)+4(1-\lambda) \mu]}{4 \mu^{2}[\lambda(1-x)+(1-\lambda) \mu]^{2}}+\frac{1}{2 \mu^{2} \lambda}=\frac{2(1-\lambda) \mu^{2}}{4 \mu^{2}[\lambda(1-x)+(1-\lambda) \mu]^{2}}>0 .
$$


We therefore concentrate on $\mu=0$. In this case, the expression reduces to:

$$
k(x ; \lambda, 0)=\frac{1}{(1-x)^{2}}\left[\frac{2-x\left(\lambda+\frac{1}{\lambda}\right)}{4 \lambda}\right]+\frac{1}{x} .
$$

Now we will argue that this expression is positive for all $x \leq \bar{x}$. This is certainly the case if $2-x\left(\lambda+\frac{1}{\lambda}\right)>0$. As the latter expression decreases in $x$, we may evaluate it at $x=\bar{x}$ to obtain $2\left(1-\frac{\lambda^{2}+1}{\lambda+1}\right) \geq 0$ and conclude $\frac{\partial \Gamma(x ; \lambda, \mu)}{\partial \lambda}>0$.

Fact $7: \Gamma(x ; 1 / 2, \mu)<0$.

Proof of Fact 7: We have

$$
\Gamma(x ; 1 / 2, \mu)=\frac{x}{(1-x)^{2}}\left(\frac{11 x-8}{8}+\frac{\ln \frac{2-x}{2 x}}{2}\right)+\frac{x}{2 \mu} \ln \left[1+\frac{\mu}{(1-x)}\right]-\frac{1}{2} .
$$

We have shown in Fact 4, step 2 that $\frac{\partial \Gamma(x ; \lambda, \mu)}{\partial \mu}<0$. Therefore $\Gamma(x ; 1 / 2,0)<0$ implies $\Gamma(x ; 1 / 2, \mu)<0$. We obtain $\Gamma(x ; 1 / 2,0)=\frac{x}{(1-x)^{2}}\left(\frac{11 x-8}{8}+\frac{\ln \frac{2-x}{2 x}}{2}\right)+\frac{2 x-1}{2(1-x)}$. We want to show that this expression is negative for $x \leq \frac{2}{3}$. This is the case iff $\frac{1}{(1-x)}\left(\frac{3 x}{8}+\frac{\ln \frac{2-x}{2 x}}{2}\right)-\frac{1}{2 x}<0$, or iff $\frac{3 x^{2}-4+4 x}{4 x}+\ln \frac{2-x}{2 x}<0$. Taking the derivative of LHS to $x$ we obtain $\frac{\partial L H S}{\partial x}=\frac{12 x^{2}+16}{16 x^{2}}-\frac{2}{x(2-x)}$. We show that this derivative is positive. This is true if $12 x^{2}+16>\frac{32 x}{2-x}$ and certainly if $12 x^{2}-32 x+16>0$. Since this polynomial has a root at $x=\frac{2}{3}$ and at $x=2$, it is positive for $x \leq \frac{2}{3}$. Therefore, the expression is increasing and we may evaluate at $x=\frac{2}{3}$ to conclude that the inequality holds.

Fact 8: For any $0<\mu<1$ and $0<x<1, \Gamma(x ; 1, \mu)>0$ and $\Gamma(1 ; 1, \mu)=$ $\Gamma(0 ; 1, \mu)=0$.

Proof of Fact 8: We obtain $\Gamma(x ; 1, \mu)=\frac{-x}{(1-x)}+\frac{x}{2(1-x)^{2}} \ln \frac{(2-x)}{x}$. This expression is positive for all $0<x<1$ if $-1+\frac{1}{2(1-x)} \ln \frac{2-x}{x}>0$ or, $\ln \frac{2-x}{x} \geq 2-2 x$. To verify the latter inequality, check that it holds with equality at $x=1$ and that $\frac{\partial L H S}{\partial x}=\frac{-2}{x(2-x)}<\frac{\partial R H S}{\partial x}=-2$, since $x(2-x)<1$.

The last equality of the statement is easily verified:

$$
\Gamma(0 ; 1, \mu)=\lim _{x \rightarrow 0} \frac{x}{2(1-x)^{2}} \ln \frac{(2-x)}{x}=0 .
$$

Fact 9: $\frac{\partial x}{\partial \mu}>0$ if $\alpha<1$.

Proof of Fact 9: We have that $\frac{d \Gamma(\cdot)}{d \mu}=\frac{\partial \Gamma(\cdot)}{\partial \mu}-\alpha \frac{\partial \Gamma(\cdot)}{\partial x}$. Above in Fact 4 (step 2) we have shown that the first term, that is, the direct effect, is negative. Furthermore, we know that in equilibrium $\frac{\partial \Gamma(\cdot)}{\partial x}>0$, therefore $\frac{\partial \Gamma(\cdot)}{\partial \mu}<0$. Now by employing the Implicit Function Theorem we obtain: $\frac{\partial x}{\partial \mu}=-\frac{\partial \Gamma(\cdot)}{\partial \mu} / \frac{\partial \Gamma(\cdot)}{\partial x}>0$. 
Fact 10: $\frac{\partial E(p)}{\partial \lambda}<0$ if $\alpha<1$ and $\frac{\partial E(p)}{\partial \lambda}=0$ if $\alpha=1$.

Proof of Fact 10: To prove the first statement, it is sufficient to show that $F(p)$ as specified in (7) increases in $\lambda$. Recall that $\frac{\partial x}{\partial \lambda}<0$ if $\alpha<1$ and consider first the lower segment $F^{l}(p)$. We can show that

$$
\frac{\partial F^{l}(p)}{\partial x}=\frac{1}{2(1-x)}\left[\frac{1}{1-x}\left(2-x-\frac{x v}{p}\right)-1-\frac{v}{p}\right]<\frac{1}{2(1-x)}\left[\frac{1}{1-x}(2-2 x)-2\right]=0 \text {, where }
$$

the inequality follows from the fact that $\frac{v}{p}>1$. Hence, $\frac{d F^{l}(p)}{d \lambda}>0$. Secondly, consider now the part of $F(\cdot)$ on the domain $\left[\lambda v, p^{\prime}\right]$. It can be checked that both terms in $\frac{d F^{m}(\cdot)}{d \lambda}=\frac{\partial F^{m}(\cdot)}{\partial \lambda}+\frac{\partial F^{m}(\cdot)}{\partial x} \frac{\partial x}{\partial \lambda}$ are positive. Finally, consider the upper segment $F^{u}(p)$. It is easily checked that $\frac{\partial F^{u}(\cdot)}{\partial x}<0$, hence $\frac{d F^{u}(p)}{d \lambda}>0$.

The second statement, $\frac{\partial E(p)}{\partial \lambda}=0$ if $\alpha=1$, can easily be verified by observing that (3) does not depend on $\lambda$.

Proposition 2: Equilibrium for the case of no channel substitution.

Proof. The proof is included in the proof of Theorem 6 .

Lemma 3: Prices in the interval $\left(\lambda v, \frac{(1-x) v}{1-x+\frac{\mu}{\lambda}-\mu}\right)$ are set with zero probability.

Proof. Let us denote the upperbound by $p^{\prime}$. To see why the lowerbound of this open interval is $\lambda v$, suppose first that a slightly higher price is asked with some probability. The firm will then not sell to the inexperienced consumers on the Internet, so it can improve by not charging that price and charging $\lambda v$ instead. Suppose now that $\lambda v$ has zero density in equilibrium and that the lowerbound of the hole is $\hat{p}<\lambda v$. We will show that firm $i$ can improve by charging $\lambda v$ with positive probability, i.e. by shifting mass from $\hat{p}$ to $\lambda v$. The profit of charging $\hat{p}$ is given by $\left(\frac{x}{2}+[1-x]\left[1-F_{j}(\hat{p})\right]\right) \hat{p}$ while the profits of charging $\lambda v$ are given by $\left(\frac{x}{2}+[1-x]\left[1-F_{j}(\lambda v)\right]\right) \lambda v$. Now since the rival $j$ has zero probability over the interval $(\hat{p}, \lambda v], F_{j}(\hat{p})=F_{j}(\lambda v)$ and thus $i$ prefers to charge $\lambda v$ in stead of $\hat{p}$. Thus, the gap consists of the interval $\left(\lambda v, p^{\prime}\right)$.

The next step is to find an expression for $p^{\prime}$ by using the fact that $F(\lambda v)=F\left(p^{\prime}\right)$. Since the firm must be indifferent between $\lambda v$ and $p^{\prime}$ it must be the case that

$$
\left(\frac{x}{2}+[1-x][1-F(\lambda v)]\right) \lambda v=\left(\frac{x}{2}+\mu\left[1-F\left(p^{\prime}\right)\right]\right) p^{\prime}
$$

From this we can obtain that

$$
1-F(\lambda v)=1-F\left(p^{\prime}\right)=\frac{\left(1-\frac{\lambda v}{p^{\prime}}\right) \frac{x}{2}}{[1-x] \frac{\lambda v}{p^{\prime}}-\mu}
$$

We know that the firm can always attain the profit $\frac{x}{2} v$, by charging $p=v$. We can use this fact to obtain an expression for $p^{\prime}$ :

$$
p^{\prime}=v \frac{1-x}{1-x+\frac{\mu}{\lambda}-\mu} .
$$


Lemma 4: Expressions for expected price and conditional expected minimum price.

Proof. The expected price can be calculated as follows:

$$
E(p)=\int_{\underline{p}}^{\lambda v} p f^{l}(p) \quad d p+\int_{p^{\prime}}^{v} p f^{u}(p) \quad d p,
$$

where $f^{l}$ and $f^{u}$ denote the density functions corresponding to the lower and upper parts of the cumulative distribution function, respectively. The first term can be derived by noting that

$$
f^{l}=\frac{d F^{l}}{d p}=\frac{x v}{(2-2 x) p^{2}}
$$

It then follows that

$$
\left.\int_{\underline{p}}^{\lambda v} f^{l} p d p=\frac{v x}{2-2 x} \ln p\right]_{\underline{p}}^{\lambda v}=\frac{x v}{2(1-x)} \ln \left[\frac{\lambda(2-x)}{x}\right] .
$$

The second term can be derived in a similar way. We have that:

$$
f^{u}=\frac{d F^{u}}{d p}=\frac{2 \mu x v}{4 \mu^{2} p^{2}}
$$

and

$$
\left.\int f^{u} p d p=\frac{x v}{2 \mu} \ln p\right]_{p^{\prime}}^{v}=\frac{x v}{2 \mu} \ln \left[1+\frac{(1-\lambda) \mu}{\lambda(1-x)}\right] .
$$

The expected price can therefore be expressed as follows:

$$
E(p)=\frac{x v}{2(1-x)} \ln \left[\frac{\lambda(2-x)}{x}\right]+\frac{x v}{2 \mu} \ln \left[1+\frac{(1-\lambda) \mu}{\lambda(1-x)}\right] .
$$

Now we turn to the minimum price. We have to determine the conditional expected value of the minimum of the two prices, that is, the expected minimum price given the fact that it is not greater than $\lambda v$. First we will derive the cumulative distribution function of the minimum price. It is defined as follows:

$$
F_{\min }(p) \equiv \operatorname{Pr}\left[\min \left[p^{1}, p^{2}\right] \leq p\right]=1-[1-F(p)]^{2} .
$$


Using $F(p)$ found above:

$$
F_{\min }(p)= \begin{cases}0 & \text { if } p<\underline{p} \\ 1-\left(\frac{x}{2(1-x)}\left[\frac{v}{p}-1\right]\right)^{2} & \text { if } \underline{p} \leq p \leq \lambda v \\ 1-\left(\frac{x(1-\lambda)}{2(1-x) \lambda}\right)^{2} & \text { if } \max [\underline{p}, \lambda v] \leq p \leq p^{\prime} \\ 1-\left(\frac{(v-p) x}{p 2 \mu}\right)^{2} & \text { if } \max \left[\underline{p}, p^{\prime}\right]<p \leq v \\ 1 & \text { if } p>v\end{cases}
$$

The inclusion of the expression $\max [\underline{p}, \lambda v]$ ensures that $F_{\min }(p)$ is nonnegative. It furthermore leads to a restriction on the domain of $x$ for the usage of the explicit expression for $F_{\min }(p)$. Consider the value $\pi \equiv F_{\min }(\lambda v)$ and note that $\underline{p}=\frac{x v}{2-x}<\lambda v$ if $x<\bar{x} \equiv \frac{2 \lambda}{\lambda+1}$. Therefore we obtain

$$
\pi=\left\{\begin{array}{c}
1-\left(\frac{x(1-\lambda)}{2(1-x) \lambda}\right)^{2} \text { if } x<\bar{x} \\
0 \text { otherwise }
\end{array}\right.
$$

The conditional expected value can be expressed as follows:

$$
E\left(\min \left[p^{1}, p^{2}\right] \mid \min \left[p^{1}, p^{2}\right] \leq \lambda v\right)=\frac{\int_{\underline{p}}^{\lambda v} p d F_{\min }(p)}{F_{\min }(\lambda v)} \text { for } x<\bar{x} .
$$

For the integral part (the numerator) we have that

$$
\begin{aligned}
\int_{\underline{p}}^{\lambda v} p \frac{d F_{\min }}{d p} d p & =\int_{\underline{p}}^{\lambda v} \frac{x^{2} v^{2}}{2(1-x)^{2} p^{2}}-\frac{x^{2} v}{2(1-x)^{2} p} d p= \\
& \left.=-\frac{x^{2} v^{2}}{2(1-x)^{2} p}-\frac{x^{2} v \ln p}{2(1-x)^{2}}\right]_{\underline{p}}^{\lambda v},
\end{aligned}
$$

and we obtain:

$$
\frac{x^{2} v}{2(1-x)^{2}}\left[\frac{-1}{\lambda}+\frac{2}{x}-1-\ln \left[\frac{(2-x) \lambda}{x}\right]\right]
$$

The conditional expected minimum price is thus:

$$
E(\min [p] \mid \min [p] \leq \lambda v)=\frac{\frac{x^{2} v}{2(1-x)^{2}}\left[\frac{-1}{\lambda}+\frac{2}{x}-1-\ln \left[\frac{(2-x) \lambda}{x}\right]\right]}{F_{\min }(\lambda v)} \text { for } x<\bar{x}
$$

Proposition 5: Equilibrium for the case of partial channel substitution.

Proof. The result is established in the text. 
Theorem 6: Characterization of equilibria.

Proof. It is clear that $x$ can range in the interval $[0,1-\mu]$. Moreover, when $\bar{x}<x \leq 1-\mu$ it has to be that $\alpha=1$. From Fact 4 we know that there are either two, one or no solutions with $x$ in the required interval to the equation $\Gamma(x ; \lambda, \mu)=0$. When there are two solutions, there is one stable equilibrium with $\alpha \in(0,1)$ and one with $\alpha=1$. When there is one solution, it follows (from Fact 1 ) that there is a unique stable equilibrium with $\alpha \in(0,1)$. Finally, when there is no solution, it has to be that there is a unique equilibrium with $\alpha=1$. We will now characterize which equilibrium situation arises when. To this end, we will fix $\mu$ at different levels and then see how the equilibrium configuration changes when $\lambda$ takes on different values.

First, consider $\mu$ close to 0 . From Facts 1 and 2 we know that at $x=0$ and $x=\bar{x}, \Gamma(x ; \lambda, \mu)<0$ for all $\lambda<1$. From Facts 6 to 8 we can then infer that there exist a $\tilde{\lambda}(\mu)$ such that for all $\lambda<\widetilde{\lambda}(\mu), \Gamma(x ; \lambda, \mu)<0$ and a unique equilibrium with $\alpha=1$ exists. When $\lambda>\widetilde{\lambda}(\mu), \Gamma(x ; \lambda, \mu)=0$ has two solutions in the interval $[0, \bar{x}]$. When $\lambda$ gets close to 1 , however, $\bar{x}$ approaches 1 and eventually becomes larger than $(1-\mu)$. Combining this with Fact 8 implies that there must be a $\widetilde{\widetilde{\lambda}}(\mu)$ such that for $\widetilde{\lambda}(\mu)<\lambda<\widetilde{\widetilde{\lambda}}(\mu)$, there exist two equilibria and for all $\lambda>\widetilde{\widetilde{\lambda}}(\mu)$ there is one stable equilibrium with $\alpha<1$.

Fix then $\mu$ close to 1 . As $x$ lies in the interval [0,1- $\mu$ ], all permissable values of $x$ lie close to 0 . From Fact 3 and the continuity of $\Gamma(\cdot)$ and $\partial \Gamma(\cdot) / \partial x$ in a neighborhood of 0 ( 0 itself excluded) it follows that we can choose $\mu$ close enough to 1 such that $\partial \Gamma(\cdot) / \partial x>0$ on the whole relevant interval. From Facts 6 to 8 it follows that there exist a $\widetilde{\lambda}(\mu)$ as defined in $(\mathrm{A})$ of the Theorem.

We next show that for any given $\mu, 0<\mu<1$, the equilibrium configurations that arise when $\lambda$ increases from 0 to 1 , are exhausted by the two possibilities considered above. From Fact 7 it follows that for $\lambda$ small enough there is only one equilibrium, namely where $\alpha=1$. From Fact 8 it follows that for $\lambda$ close to 1 , there exist a unique stable equilibrium with $\alpha<1$. Finally, from Fact 6 it follows that if for $(\widehat{\lambda}, \mu)$ there exists a unique stable equilibrium with $\alpha<1$, then this is also the case for any $(\lambda, \mu)$ with $\lambda>\widehat{\lambda}$. Hence, no other possibilities exist than the two considered above.

We finally show that the existence of $\bar{\mu}$ as defined in the Theorem. To this end we show that if for a certain $\widehat{\mu}$ the equilibrium configuration for increasing $\lambda$ shifts from a unique equilibrium with $\alpha=1$, via two stable equilibria to a unique equilibrium with $\alpha<1$, then this pattern has to exist for all $\mu<\widehat{\mu}$. In the proof of Fact 4 we show that $\partial \Gamma(\cdot) / \partial \mu<0$. Moreover, the smaller $\mu$, the larger the range of values for which $\Gamma(x ; \lambda, \mu)$ is defined. This implies, together with Facts 1 and 2 , that if $(\lambda, \widehat{\mu})$ is in the region where there are two equilibria, then $(\lambda, \mu)$ is also in this region for any $\mu<\widehat{\mu}$. Moreover, Facts 7 and 8 are true for any value of $\mu$ so that the existence of $\widetilde{\lambda}(\mu)$ and $\widetilde{\widetilde{\lambda}}(\mu)$ is guaranteed.

The observation that $\lim _{\lambda \rightarrow 1} \underline{\mu}(\lambda)=0$ and $\lim _{\lambda \rightarrow 1} \bar{\mu}(\lambda)=1$ in Proposition 2 
follows from Fact 8 .

\section{References}

[1] Ulf Andersen: "Global e-Commerce Report, 2000", Taylor Nelson Sofres, www.tnsofres.com.

[2] Joseph Bailey: "Electronic Commerce: Prices and Consumer Issues for three Products: Books, Compact Discs and Software", Organization for Economic Co-operation and Development, OCDE/GD(1998)4.

[3] Yannis Bakos: "Reducing Buyer Search Costs: Implications for Electronic Marketplaces", Management Science 43-12, 1976-93, 1997.

[4] Boston Consulting Group: "The race for online riches, E-retailing in Europe", www.bcg.com, February 2000.

[5] Eric Brynjolfsson and Michael Smith: "Frictionless Commerce? A comparison of Internet and Conventional Retailers", Management Science 46-4, 563-585, 2000.

[6] Karen Clay, Ramayya Krishnan, Eric Wolff and Danny Fernandes: "Retail Strategies on the Web: Price and Non-Price Competition in the Online Book Industry", Carnegie Mellon University, mimeo, 2000.

[7] Joseph E. Harrington, Jr.: "Comment on 'Reducing Buyer Search Cost: Implications for Electronic Marketplaces' (Management Science, 1997)", The Johns Hopkins University, mimeo, 2001.

[8] Arno Hummerston: "Global e-Commerce Report, 2001", Taylor Nelson Sofres, www.tnsofres.com.

[9] Maarten Janssen and José Luis Moraga: "Pricing, Consumer Search and the Size of Internet Markets" Tinbergen Institute Discussion Paper, May 2000.

[10] Rajiv Lal and Miklos Sarvary: "When and How is the Internet Likely to Decrease Price Competition?" Management Science 18, 485-503, 1999.

[11] Ho Geun Lee: "Do Electronic Marketplaces Lower the Price of Goods?", Communications of the ACM 41-12, 1997.

[12] Cristina Mazón and Pedro Pereira: "Electronic Commerce, Consumer Search and Retailing Cost Reduction", mimeo, Universidad Complutense de Madrid, December 2000. 
[13] Florian Zettelmeyer: "Expanding to the Internet: Pricing and Communications Strategies When Firms Compete on Multiple Channels", Journal of Marketing Research August, 292-308, 2000. 
Figure A1: Characterization of Equilibria

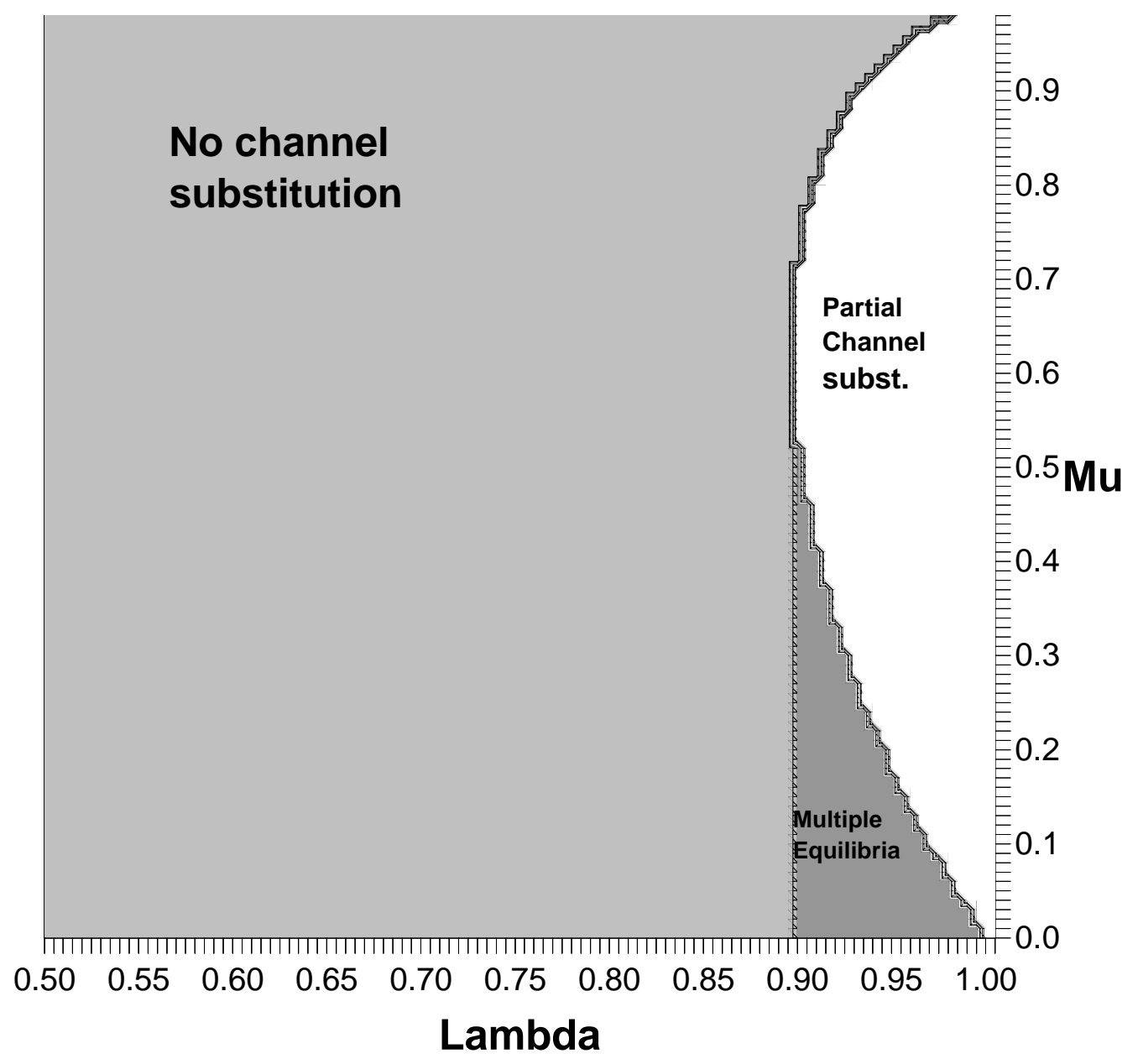


Figure A2: Sales in conv channel $(X)$ and Expected Price, $M u=0.3$

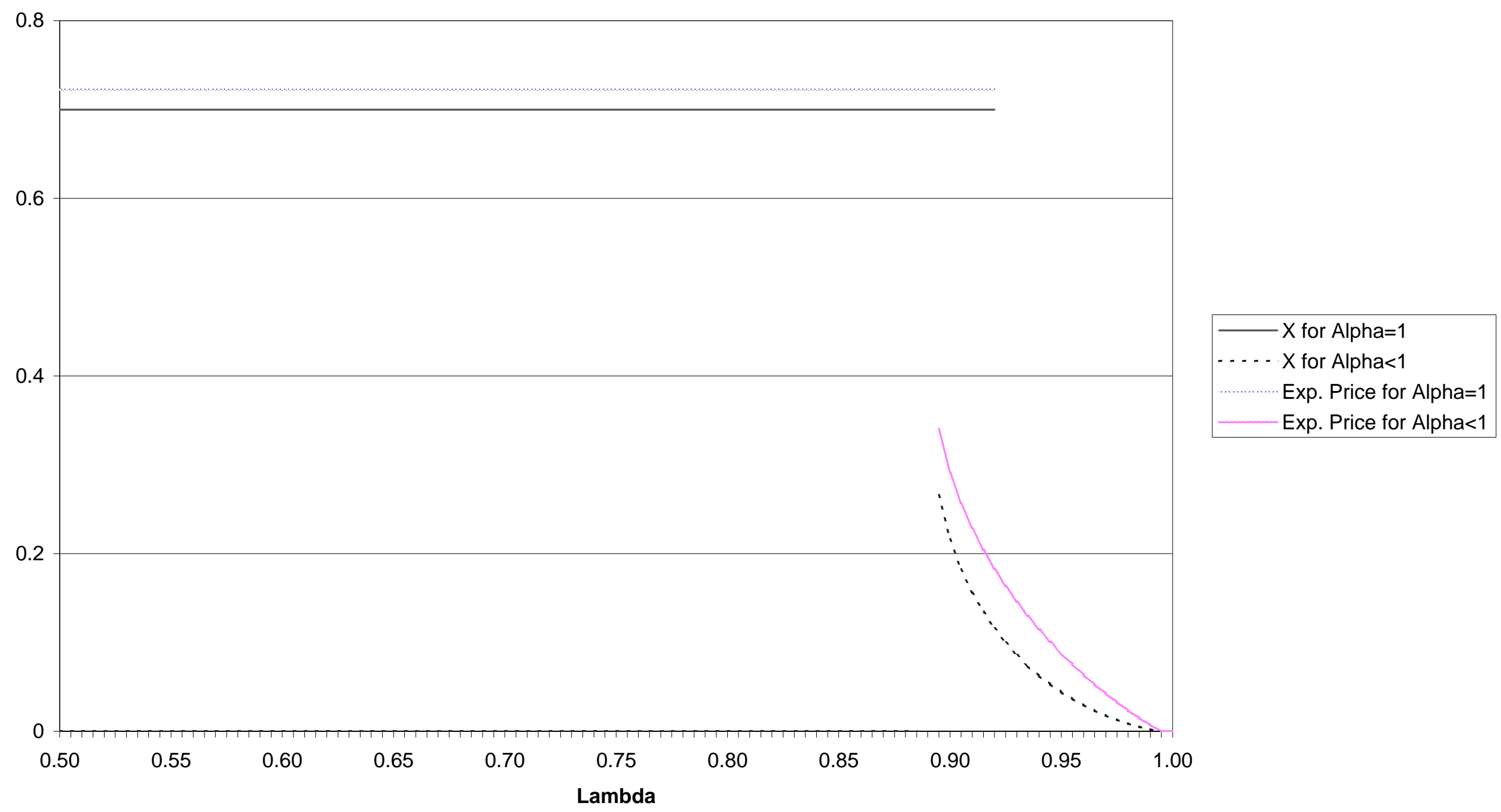


Figure A3: Sales in conv channel $(X)$ and Expected Price, Lambda $=0.9$

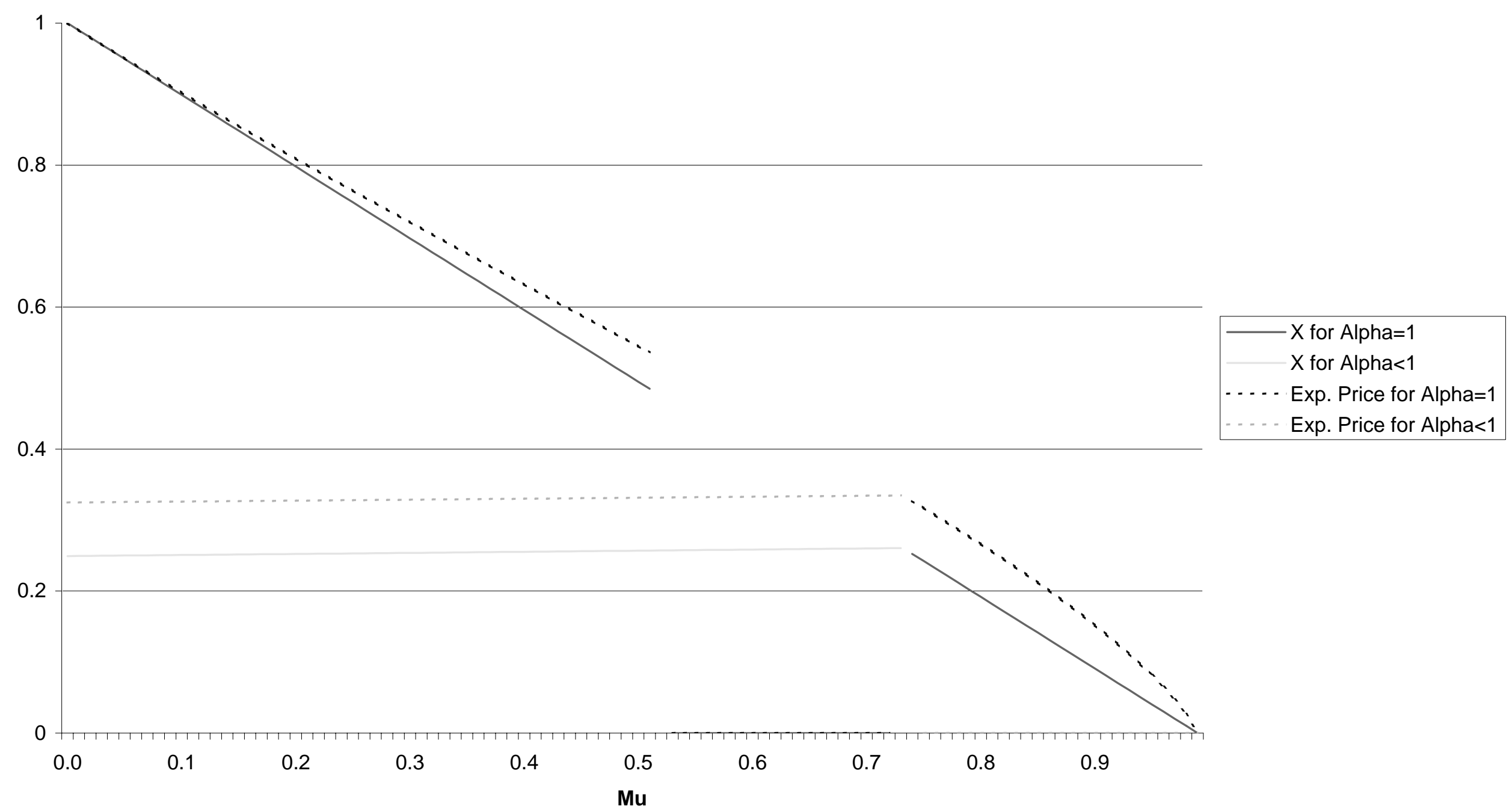


Table T1: Expected Price for different values of $\lambda$ and $\mu$.

\begin{tabular}{|c|c|c|c|c|c|}
\hline \multicolumn{6}{|c|}{ Expected price $^{\top}$} \\
\hline$\mu$ & $\lambda=0.80$ & $\lambda=0.85$ & $\lambda=0.90$ & $\lambda=0.95$ & $\lambda=0.99$ \\
\hline 0.1 & 0.903018 & 0.903018 & \begin{tabular}{|ll}
0.903018 & 0.325928 \\
\end{tabular} & $\begin{array}{ll}0.903018 & 0.099393 \\
\end{array}$ & 0.007025112 \\
\hline 0.2 & 0.810930 & 0.810930 & $\begin{array}{|ll|}0.810930 & 0.327219 \\
\end{array}$ & 0.099400 & 0.007025113 \\
\hline 0.3 & 0.722212 & 0.722212 & 0.7222120 .328531 & 0.099406 & 0.007025113 \\
\hline 0.4 & 0.635473 & 0.635473 & 0.6354730 .329865 & 0.099413 & 0.007025114 \\
\hline 0.5 & 0.549306 & 0.549306 & $0.549306 \quad 0.331223$ & 0.099419 & 0.007025115 \\
\hline 0.6 & 0.462098 & 0.462098 & 0.332608 & 0.099426 & 0.007025115 \\
\hline 0.7 & 0.371700 & 0.371700 & 0.334021 & 0.099432 & 0.007025116 \\
\hline 0.8 & 0.274653 & 0.274653 & 0.274653 & 0.099438 & 0.007025117 \\
\hline 0.9 & 0.163580 & 0.163580 & 0.163580 & 0.099445 & 0.007025117 \\
\hline 0.99 & 0.026734 & 0.026734 & 0.026734 & 0.026734 & 0.007025118 \\
\hline
\end{tabular}

${ }^{1}$ For $v=1$. The white entries refer to no channel substitution and the grey entries refer to partial channel substitution. 